\title{
An Optimization-Based Framework for the Identification of Vulnerabilities in Electric Power Grids Exposed to Natural Hazards
}

\author{
Yiping Fang, ${ }^{1}$ Giovanni Sansavini, ${ }^{2 *}$ and Enrico $\mathrm{Zio}^{3,4}$ \\ ${ }^{1}$ Chaire Systems Science and the Energy Challenge, Fondation Electricité de France (EDF), \\ Laboratoire Génie Industriel, CentraleSupélec, Université Paris-Saclay, Gif-sur-Yvette, France \\ ${ }^{2}$ Reliability and Risk Engineering Laboratory, Institute of Energy Technology, Department of \\ Mechanical and Process Engineering, ETH Zurich, Switzerland \\ ${ }^{3}$ Mines ParisTech, PSL Research University, CRC, Sophia Antipolis, France \\ ${ }^{4}$ Energy Department, Politecnico di Milano, Italy
}

\begin{abstract}
This paper proposes a novel mathematical optimization framework for the identification of the vulnerabilities of electric power infrastructure systems (which is a paramount example of critical infrastructure) due to natural hazards. In this framework, the potential impacts of a specific natural hazard on an infrastructure are firstly evaluated, in terms of failure and recovery probabilities of system components; these are, then, fed into a bi-level attacker-defender interdiction model to determine the critical components whose failures lead to the largest system functionality loss. The proposed framework bridges the gap between the difficulties of accurately predicting the hazard information in classical probability-based analyses and the over-conservatism of the pure attacker-defender interdiction models. Mathematically, the proposed model configures a bi-level max-min mixed integer linear programming (MILP) that is challenging to solve. For its solution, the problem is casted into an equivalent one-level MILP that can be solved by efficient global solvers. The approach is applied to a case study concerning the vulnerability identification of the georeferenced RTS24 test system under simulated wind storms. The numerical results demonstrate the effectiveness of the proposed framework for identifying critical locations under multiple hazard events and, thus, for providing a useful tool to help decision-makers in making more-informed pre-hazard preparation decisions.
\end{abstract}

KEY WORDS: vulnerability analysis; critical infrastructures; natural hazards; network interdiction; attacker-defender interdiction model; mathematical optimization

\footnotetext{
* Address correspondence to Giovanni Sansavini, ETH Zurich, Leonhardstrasse 21, 8092 Zurich, Switzerland, Tel.: +41446325038; sansavig@ethz.ch
} 


\section{INTRODUCTION}

Modern society relies on the effective functioning of critical infrastructure (CI) systems such as the power grid, transportation network, Internet, water distribution network, etc. to provide public services, improve quality of life, sustain private profits and spur economic growth. Recent years have seen many disruptions of CIs caused by natural disasters (i.e., floods, ice and wind storms, hurricanes, tsunamis and earthquakes) leading to a substantial impact on the human livelihoods and economic properties (Munich, Kron, \& Schuck, 2014). Furthermore, there is a significant concern that the number and severity of these extreme natural events will increase in the future as a result of global warming and climate changes (Cutter et al., 2015). Hence, there is a need of techniques and tools to assess the impact of extreme natural events on CIs, in support policymakers and investments in system protection practices (Cadini, Agliardi, \& Zio, 2017a, 2017b; Rocchetta, Li, \& Zio, 2015).

A key component of the protection of CIs against natural disasters for managing and mitigating service disruptions is the ability to evaluate potential vulnerabilities. Vulnerability analysis for CI systems has been given increased attention in the research community during the last decades. Different definitions and pre-analytic visions of vulnerability have been developed by diverse researchers and policy makers from different knowledge domains. As a result, the implementation of vulnerability analysis takes different forms (Johansson, Hassel, \& Zio, 2013). For example, Haimes (2006) defines vulnerability as "the manifestation of the inherent states of the system that can be exploited to adversely affect that system"- stressing that vulnerability is concerned with the intrinsic characteristics of a system rather than the environment in which the system is located. Aven (2011) interprets vulnerability as the uncertainty about and severity of the consequences of the activity given the occurrence of the accident initiating event, which is scenario-specific. As a matter of fact, albeit vulnerability is often viewed as an inherent characteristic of a system, most researchers acknowledge that vulnerability is conditional on a hazard or that it is useless to discuss vulnerability independent of its hazard context (Birkmann, 2007). Overall, the concept of vulnerability has been continuously widened and broadened towards a more comprehensive vision, and interested readers can refer to some relevant discussions and overviews on this in the literature (Ezell, 2007; Füssel, 2007; Kröger \& Zio, 2011; Murray \& Grubesic, 2007; Scholz, Blumer, \& Brand, 2012; Zio, 2016; Zio \& Aven, 2011).

In the context of the present paper, we follow previous studies (Apostolakis \& Lemon, 2005; Ezell, 2007) and view vulnerability as a measure of system susceptibility to scenarios for more narrowly identifying weak points in the system within the context of a scenario. Thus, vulnerability analysis is adopted from the perspective of critical components analysis that focuses on the identification of important components or combinations of components with regard to the impact on system functionality loss subject to a natural hazard (Jönsson, Johansson, \& Johansson, 2008; Oh, Deshmukh, \& Hastak, 2012; Zio \& Sansavini, 2011, 2013). In this paper, we carry out the vulnerability analysis in support to 
short-term pre-event preparation practices, e.g. choosing critical power poles to be hardened or allocating backup power units in an electrical power system before a specific typhoon strikes the system.

A range of approaches have been proposed in the literature for the vulnerability assessment of CI systems under natural hazards (Zio \& Kroger, 2009). Sohn (2006) used an accessibility perspective to study the vulnerability of a highway network under flood damage by evaluating the significance of its links. Dawson, Peppe, and Wang (2011) proposed a multi-agent simulation method coupled with a hydrodynamic model to evaluate the vulnerability of individuals to flooding threats. Jenelius and Mattsson (2012) presented a grid-based approach to analyze the vulnerability of road networks under area-covering disruptions. The road network is covered using a grid of uniformly shaped and sized cells, where each cell represents the spatial extent of a disrupting event. Adachi and Ellingwood (2008) introduced a probability-based simulation method to study the seismic vulnerability of a municipal water system taking into account the supporting electrical power system. Na and Shinozuka (2009) applied a similar simulation-based method for the seismic loss estimation of seaport transportation systems. Hong, Ouyang, Peeta, He, and Yan (2015) proposed a comprehensive methodology to quantitatively assess the railway system vulnerability under floods using historical data and GIS technology. Other important investigations concerning the vulnerabilities of various CI systems under natural hazards include the seismic vulnerability analysis of the power grid and water pipeline system in Shelby County, USA (Hernandez-Fajardo \& Dueñas-Osorio, 2011, 2013), and of the European gas and electricity systems (Poljanšek, Bono, \& Gutiérrez, 2012), the vulnerability assessment of the power grid and gas network under wind storms (Ouyang \& Dueñas-Osorio, 2014; Salman, Li, \& Stewart, 2015; Winkler, DuenasOsorio, Stein, \& Subramanian, 2010), the vulnerability of telecommunication systems to hurricanes (Kwasinski, 2010) and the lightning vulnerability of the power grid (Dueñas-Osorio \& Vemuru, 2009).

The above vulnerability studies analyze different CI systems and different types of natural hazards, but generally entail a probability-based analysis framework, which includes the following steps: i) threat characterization by modeling the specific natural hazard, ii) estimation of failure probabilities of system components under the hazard scenario, iii) simulation of the damage state of each component, and iv) modeling and analysis of the system functional response given the component damages. This simulationbased analysis framework is valuable for assessing system vulnerability in a statistical manner, i.e., computing the average system performance loss or identifying the critical components in the system, based on different realizations of a specific hazard. However, for a specific realization/estimation of a hazard event, the uncertainty within the estimated failure probabilities can be propagated by the simulation-based methods, leading to underestimation or overestimation of system vulnerability. Actually, it is extremely difficult to accurately predict the failure probabilities of each components in a CI system exposed to a natural hazard. Therefore, there is a strong need to develop more robust tools to assist decision makers during pre-hazard preparation (Pidgeon, 2012). 
Optimization methods have been applied for the identification of weak locations vital to the operation of network systems. Interdiction models have been developed to assess the vulnerabilities of network systems based on the component importance to system functionality (Arroyo, 2010; Bier, Gratz, Haphuriwat, Magua, \& Wierzbicki, 2007; G. Brown, Carlyle, Salmeron, \& Wood, 2005; Church, Scaparra, \& Middleton, 2004; Delgadillo, Arroyo, \& Alguacil, 2010; Matisziw \& Murray, 2009; Matisziw, Murray, \& Grubesic, 2007; Ramirez-Marquez, 2010; Salmeron, Wood, \& Baldick, 2004; Wood, 2011). In the classical problem of network interdiction, an intelligent attacker's activities are modeled using the constructs of network optimization (e.g., maximum flows, multi-commodity flows, and shortest paths), and attacks target the network's components to disrupt the network's functionality to the maximum, resulting in a bi-level attacker-defender Stackelberg game in a mathematical form of "max-min" (or "min-max") programming (Wood, 2011). Its extension to trilevel defender-attackerdefender system defense models in supporting the allocation of limited protection resources have also been studied in CI protection planning (G. Brown, Carlyle, Salmerón, \& Wood, 2006; Y.-P. Fang \& Zio, 2019; Y. Fang \& Sansavini, 2017; Ouyang \& Fang, 2017). By exploiting optimization, these interdiction models intend to establish bounds for network vulnerability in terms of critical components associated with worst-case impacts to system performance. In other words, these methods might overestimate the system performance loss during the vulnerability analysis of a CI system under a natural hazard, and the identified critical components may not necessarily be failed by the hazard.

To overcome the drawbacks of the aforementioned methods, this paper presents a novel optimization-based mathematical framework for the identification of the vulnerabilities of electric power infrastructure systems (which is a paramount example of CI) under natural hazards by combining the interdiction models and the predicted information of specific natural hazards. In particular, the timevarying failure probabilities of system components are firstly computed by integrating the spatialtemporal profile of the natural hazard and the structural fragilities of the components. The restoration time of components is also estimated probabilistically. Then, the attacker-defender interdiction game is modeled as an optimization problem, which incorporates the probabilities of failure and restoration of the components, and identifies critical parts of the system. Therefore, the failure scenarios identified by the optimization represent the most-likely worst cases under the specific hazard. The proposed approach bridges the gap between the difficulties of accurately predicting the hazard information in the classical probability-based analyses and the over-conservativeness of the pure attack-defender interdiction models for CI vulnerabilities analysis under a specific natural hazard, thus, providing a useful tool to help decision-makers in making more-informed pre-hazard preparation decisions.

The remainder of the paper is organized as follows. Section 2 introduces the models for evaluating the impacts of natural hazards on CI systems, including threat characterization, structural fragility and component restoration time models. In Section 3, the detailed formulation of the optimization framework for the identification of CI vulnerabilities is proposed. Section 4 proposes the solution methodology for the proposed optimization model. Section 5 presents the numerical results by applying 
the proposed framework to the georeferenced RTS24 power test system. Relevant discussion and concluding remarks are provided in Section 6.

\section{IMPACT OF NATURAL HAZARDS ON CRITICAL INFRASTRUCTURES}

Depending on the nature of the formation process, natural disasters can be divided into: geophysical (earthquake, volcano, and tsunami), meteorological (tropical storm, tornado, blizzard, ice storm, and drought), and hydrological (flood), biological (epidemics and insect pests), and extraterrestrial (meteor). The former three types are generally most destructive to CI systems. They include not just one single instantaneous impact, but multiple and even continuous impacts. For instance, the windstorms that affected China in 2005 caused more than 60 high-voltage power transmission towers to collapse, and the ice and snow storms that devastated a large area in South China lasted for hours (Xie \& Zhu, 2011). Disasters can even last for days, like the hurricane Sandy (2012) in the United States, where many of the CIs were wiped out in most of the eastern US (especially the coastal Mid-Atlantic States). Moreover, hazard impacts often are difficult to characterize because a given natural hazard may initiate a number of different threats. For example, tropical storms can cause damages through wind, rain, storm surge and islanding flooding. The most significant characteristics for assessing the disaster impacts are speed, onset, availability of perceptual cues (such as wind, rain, or ground movement), intensity, scope and duration of impact (Lindell \& Prater, 2003). Table I summarizes the basic characteristics of natural disasters (Guikema, Davidson, \& Liu, 2006; Wang, Chen, Wang, \& Baldick, 2016).

Table I. Characteristics of natural disasters

\begin{tabular}{|c|c|c|c|c|}
\hline Disaster type & Impact region & Predictability & Span/Area & Affecting time \\
\hline $\begin{array}{l}\text { Tropical storm } \\
\text { hurricane }\end{array}$ & Coastal regions & $\begin{array}{l}24-72 \text { hours, } \\
\text { moderate to good }\end{array}$ & $\begin{array}{l}\text { Large (radius up } \\
\text { to } 1500 \mathrm{~km} \text { ) }\end{array}$ & Hours to days \\
\hline Tornado & Inland plains & $\begin{array}{l}0 \text { - } 2 \text { hours, bad to } \\
\text { moderate }\end{array}$ & $\begin{array}{l}\text { Small (radius up } \\
\text { to } 8 \mathrm{~km} \text { ) }\end{array}$ & $\begin{array}{l}\text { Minutes to } \\
\text { hours }\end{array}$ \\
\hline $\begin{array}{l}\text { Blizzard, ice } \\
\text { storm }\end{array}$ & $\begin{array}{l}\text { High latitude } \\
\text { regions }\end{array}$ & $\begin{array}{l}24-72 \text { hours, } \\
\text { moderate to good }\end{array}$ & $\begin{array}{l}\text { Large (up to } \\
1500 \mathrm{~km} \text { ) }\end{array}$ & Hours to days \\
\hline Earthquake & $\begin{array}{l}\text { Regions on fault } \\
\text { lines }\end{array}$ & $\begin{array}{l}\text { Seconds to } \\
\text { minutes, bad }\end{array}$ & Small to large & $\begin{array}{l}\text { Minutes to days } \\
\text { (aftershock) }\end{array}$ \\
\hline Tsunami & Coastal regions & $\begin{array}{l}\text { Minutes to hours, } \\
\text { moderate }\end{array}$ & Small to large & $\begin{array}{l}\text { Minutes to } \\
\text { hours }\end{array}$ \\
\hline $\begin{array}{l}\text { Drought, Wild } \\
\text { fire }\end{array}$ & Inland regions & Days, good & Medium to large & Days to months \\
\hline Flooding & $\begin{array}{l}\text { Low-lying } \\
\text { regions }\end{array}$ & Moderate to good & Small to large & Days to months \\
\hline
\end{tabular}

Physical impacts of natural disasters on CIs vary substantially across different hazard types and CI systems. The prediction and evaluation of the impacts are challenging tasks due to the presence of 
uncertainty about the highly dynamic evolution of hazards, as well as the inherent complexity of largescale CI systems. In the remaining part of this section, we introduce how the impacts of a specific type of natural hazard, i.e., wind storms, on components of power systems can be analyzed through the combination of threat characterization, fragility models of system components and system restoration models.

\subsection{Threat Characterization}

The primary step to evaluate the impacts of a specific natural hazard on a CI system is to model the spatiotemporal profile of all the threats engendered from the hazard because CI systems cover extensive geographic scales (Kröger \& Zio, 2011; Zio, 2016). Threat characterization models aim to associate the forecasted hazard parameters with the estimation of local threat intensity for each CI components.

A wind storm (typhoon or hurricane) event is represented by its key information forecasted, e.g. landing time and positions, approaching angle, translational velocity, central pressure difference, maximum wind speed, radius of maximum wind, which can be obtained through climate models (CMs) and/or real measurement data (Davis et al., 2008). The majority of wind-storm-related power outages in power transmission happens because trees are blown onto power lines and poles, and/or high intense winds directly blow down poles during storms (Han, Guikema, \& Quiring, 2009). Thus, the intensity of wind is regarded as the primary threat of storms. The wind speeds profile for a storm can be generated through parametric radial wind field models (Batke, Jocque, \& Kelly, 2014; Davis et al., 2008; Holland, 1980; Holland, Belanger, \& Fritz, 2010). The wind speed at location $(x, y)$ at time $t$ can be represented by (Holland et al., 2010)

$$
v(x, y ; t)=v_{m}\left\{\left(\frac{R_{m}}{r}\right)^{b} e^{\left[1-\left(\frac{R_{m}}{r}\right)^{b}\right]}\right\}^{a}
$$

where $r$ is the distance from the point to the storm center $\left(x_{\text {center }}(t), y_{\text {center }}(t)\right)$, which moves in the translational velocity $v_{t}$ of the storm, $v_{m}$ is the maximum wind speed, $R_{m}$ is the radius of maximum wind (also called as wind radius) and can be calculated from the storm eye-diameter (ED) (Batke et al., 2014), $b$ is the empirical Holland parameter and can be estimated based on the central pressure of the storm, and $a$ is a scaling parameter that adjusts the wind profile shape and a value of $a=0.5$ is typically used (Holland et al., 2010). Fig. 1 shows an example of wind profile of the Typhoon Meranti at 2016 September 14, 18:00 (GMT+8) when making landfall at Xiamen, China, calculated by Eq. (1) based on the dataset from the National Oceanic and Atmospheric Administration (NOAA) of the United States (NOAA, 2016).

Structural damage is mostly related to peak gust wind speed, which is measured as the largest speed during a specified period (usually 3 seconds). A gust factor can be used to convert the surface wind speed calculated by Eq. (1) to the most likely peak gust speed. A gust model has been developed for 
modeling gust factors (Vickery \& Skerlj, 2005), and a justified empirical value of 1.287 can be used ( $\underline{\text { Xu \& Brown, 2008). }}$.

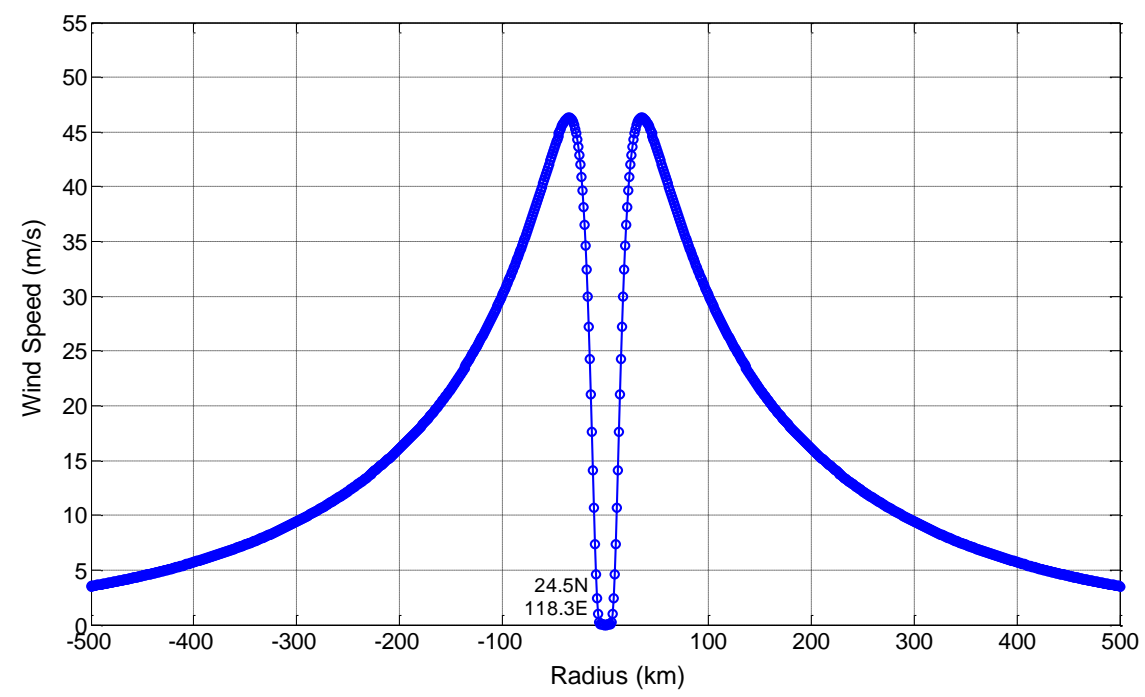

Fig. 1. Wind profile of the Typhoon Meranti at 2016 September 14, 18:00 (GMT+8) when making landfall at Xiamen, China

Storm-induced flooding is not considered here as a major threat to power systems, though storm surges associated with landfalling wind storms can cause damages to underground power components and substations (R. Brown, 2009). Yet, detailed threat models of storm flooding considering local geospatial information exist in the literature (Aerts, Lin, Botzen, Emanuel, \& de Moel, 2013; Lin, Emanuel, Oppenheimer, \& Vanmarcke, 2012), and they can be included if relevant data are available.

\subsection{Structural Fragility Models}

The functionality state of each components within a CI system can be determined by the following three steps: i) identify the key (types of) components of the system, ii) modeling the fragility of components, and iii) failure probability assignment.

In the first step, the types of components identified vulnerable to the threat, whose failures could possibly have a high impact on system performance, are identified. Although power systems comprise many types of components, it is practical to focus on the most important ones, e.g. substations and overhead lines (including the support structures and the conductors between structures). As a matter of fact, power outages due to storm-type events are most often a result of damage to overhead power lines caused by strong winds (Campbell, 2012). Therefore, in this study, we assume that generation and substation plants are not directly affected by the windstorm and consider only the failures of overhead lines, although generation nodes can be disconnected due to outages of transmission corridors.

Fragility analysis is required to compute the probability of failure of components under certain levels of threat intensity. The concept of fragility curves originates from structural reliability analysis (Booker, Torres, Guikema, Sprintson, \& Brumbelow, 2010; Espinoza, Panteli, Mancarella, \& Rudnick, 2016; Y. 
Li \& Ellingwood, 2006), and, represents the conditional probability of failure of a structural element as a function of disaster strength parameters like wind speed and precipitation, as illustrated in Fig. 2.

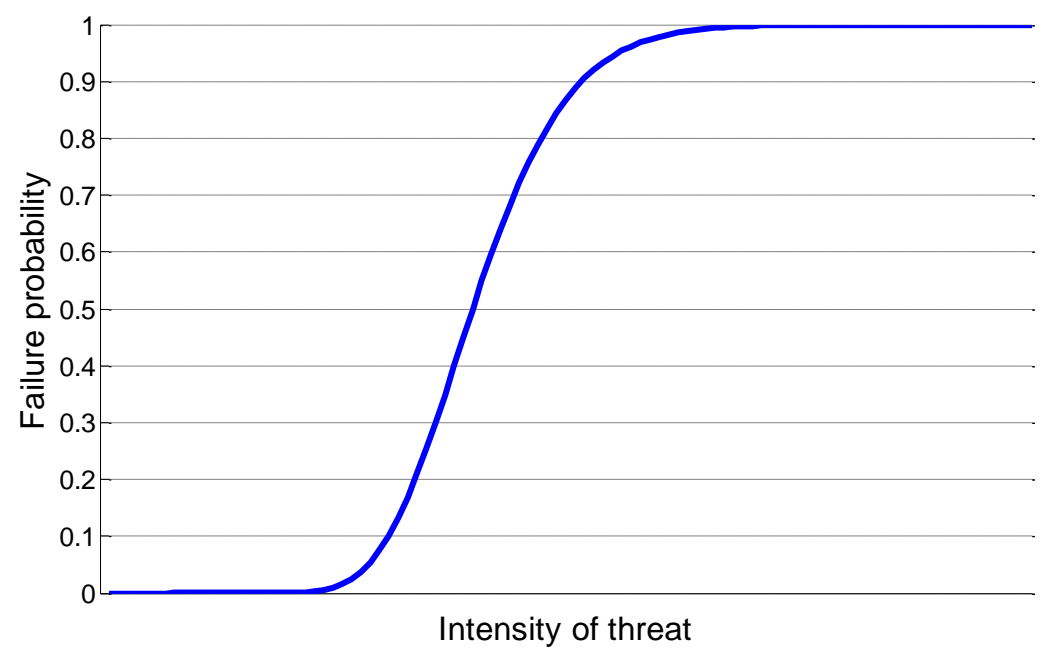

Fig. 2. Generic fragility curve

The calculation of fragility curves is often based on parametric statistical models, taking into account factors like the designed strength and aging of the components. For different CI components, different fragility curves may be used as best fits to historical data. Regarding power systems, there is a range of literature discussing the structural fragility models subject to wind loading (Bjarnadottir, Li, \& Stewart, 2012; Fenton \& Sutherland, 2011; Hangan, Savory, El Damatty, Galsworthy, \& Miller, 2008; Salman et al., 2015; Savory, Parke, Zeinoddini, Toy, \& Disney, 2001). The lognormal distribution is usually assumed to describe the fragility curves of support poles and overhead power lines (Bjarnadottir et al., 2012; Salman et al., 2015), and the direct threat-induced failure probability $p(v(t))$ as a function of the wind speed $v(t)$ is given by the following lognormal cumulative distribution function (CDF)

$$
p(v(t))=\Phi\left[\frac{\ln (v(t) / m)}{\sigma}\right]
$$

where $\Phi(\cdot)$ is the CDF of the standard normal distribution, $m$ is the median of the fragility function, and $\sigma$ is the logarithmic standard deviation of the intensity measure. The values of the parameters $m$ and $\sigma$ are related with the structural characteristics of the component under consideration.

In the third step, the overall failure probability of each component is computed by taking into account direct and indirect threats that could lead to failure. For example, besides failures caused by direct wind load, overhead power lines also fail due to falling trees and flying debris. Actually, around $55.2 \%$ of power outages in the U.S. Northeast regional distribution systems are caused by trees falling down during wind storms (G. Li et al., 2014). In addition, overhead lines consist of support poles, conductor wires and other types of equipment (Ouyang \& Dueñas-Osorio, 2014). The collapse of a single pole or conductor results in the disconnection of the entire line. Therefore, the overall failure probability of an overhead line is modeled as a series system with the fragility analysis of each pole and conductor 
associated with that line. It is assumed that the fragility of different components of an overhead line is independent. The overall failure probability of an overhead line $l$ under wind speed $v(t)$ is calculated as

$$
p_{l, \text { failure }}(v(t))=1-\prod_{k=1}^{m}\left[1-p_{S_{k}}(v(t))\right] \prod_{k=1}^{n}\left[1-p_{C_{k}}(v(t))\right]
$$

where $m$ is the number of poles supporting line $l, n$ is the number of conductor lines between two adjacent poles at line $l, p_{S_{k}}$ is the conditional failure probability of the $k$ th pole at line $l$ which can be given by Eq. (2), and $p_{C_{k}}$ is defined as the failure probability of conductor $k$ between two poles. This probability can be modeled by (Ouyang \& Dueñas-Osorio, 2014; Winkler et al., 2010):

$$
p_{C_{k}}(v(t))=\max \left(p_{C_{k}, w}(v(t)), \varpi p_{C_{k}, w t}(v(t))\right)
$$

where $p_{C_{k}, w}(v(t))$ is the direct wind-induced failure probability of conductor $k ; p_{C_{k}, w t}(v(t))$ represents the fallen tree-induced failure probability of conductor $k$; and $\varpi$ is the average tree-induced failure probability of overhead conductors, reflecting the efforts of trimming trees of utilities and assumed constant (Ouyang \& Dueñas-Osorio, 2014). The direct wind-induced failure probability $p_{C_{k}, w}(v(t))$ can be computed by Eq. (2), based on the structure property parameters of the conductor (Bayliss, Bayliss, \& Hardy, 2012). The fallen tree-induced failure probability $p_{C_{k}, w t}(v(t))$ can be calculated approximately as (Canham, Papaik, \& Latty, 2001)

$$
\log \left(\frac{p_{C_{k}, w t}(v(t))}{1-p_{C_{k}, w t}(v(t))}\right)=a_{s}+c_{S}\left(k_{z} S_{k}\right) D_{H}^{b_{S}}
$$

where $a_{s}, b_{s}$, and $c_{s}$ are parameters related with tree species, $S_{k}$ the wind intensity (0-1 scale) at the conductor, and $D_{H}$ the tree diameter at breast height. The parameter $S_{k}$ can be calculated by dividing the local peak gust wind speed by the maximum wind speed in the affected area (Canham et al., 2001).

\subsection{Component Restoration Time Model}

A range of models have been proposed in the literature for the post-disaster restoration processes of various CI systems (Duffey \& Ha, 2013; Guikema, Quiring, \& Han, 2010; Liu, Davidson, \& Apanasovich, 2007; Nateghi, Guikema, \& Quiring, 2011). The output of these models is usually represented by restoration curves at the system level (percentage of customers with service versus time) or by system average interruption duration indices (SAIDI). Yet, for system criticality analyses aiming at supporting pre-event decision making, models for estimating the restoration times of components are required. The response to the disaster and the restoration time of failed CI components varies directly with: (i) storm categories, (ii) locations and types of damaged components, and (iii) the amount of repair crews and material resources available. Thus, the restoration time of a failed component can be expressed by 


$$
T=f(\text { category, location, type, resources). }
$$

In practice it is usually challenging to have an analytic form of $f(\cdot)$. Instead, probabilistic models like Gaussian (Ouyang \& Dueñas-Osorio, 2014) and exponential distributions (Espinoza et al., 2016; Zapata, Silva, Gonzalez, Burbano, \& Hernandez, 2008) are traditionally used to represent the repair processes of power system components. Zapata et al. (2008) studied realistic historical data and showed that the lognormal distribution is a more appropriate model for component repair times in power systems. On the other hand, storm categories and intensities significantly affect the repair times of the damaged components, e.g., more time is needed for the repair crews to approach safely the affected areas under severe weather conditions. This effect can be modeled as an increase in the Mean Time To Repair $(M T T R)$ of components by a factor of restoration stress (RS). For example, Espinoza et al. (2016) assumed random RS values in the range $\{2,4\}$ for overhead lines restoration under moderate storms. In practice, data about RS can be obtained or estimated from past repair experience under different storm categories (Bhuiyan \& Allan, 1994).

Therefore, for a given storm category, the probability that a failed component, e.g., an overhead line $l$, is repaired within time $T$ is given by (Zapata et al., 2008)

$$
p_{l, \text { repair }}(\tau \leq T \mid \text { catg })=\Phi\left\{\frac{\ln \left[T /\left(R S_{c a t g} \cdot M T T R_{l}\right)\right]}{\sigma}\right\}
$$

where $R S_{\text {catg }}$ represents the restoration stress under storm category catg, $M T T R_{l}$ is the MTTR of overhead line $l$ under normal operation, and $\sigma$ is the logarithmic standard deviation of restoration time.

\section{MATHEMATICAL FORMULATION OF THE OPTIMIZATION MODEL}

For conducting the system vulnerability analysis, the failure probabilities of the CI components obtained from the hazard model and component fragility models can be fed to simulation-based models, e.g., the Sequential Monte Carlo-based time-series simulation (Espinoza et al., 2016; Kadri, Birregah, \& Châtelet, 2014; Lindell \& Prater, 2003). However, the uncertainty of the estimated failure probabilities can be propagated by simulation-based methods and lead to underestimation or overestimation of system vulnerability, especially for a specific realization of hazard event. On the other hand, the vulnerabilities of $\mathrm{CI}$ can be identified by a worst-case interdiction analysis, i.e., by an attackerdefender bi-level programming model (Arroyo, 2010; G. Brown et al., 2005; Y. Fang \& Sansavini, 2017; Salmeron et al., 2004). Nevertheless, the pure attacker-defender approach does not take into account the predicted information of specific natural hazards, as well as the spatiotemporal correlations of the natural hazards which strongly impact the probability of some common cause failures. Therefore, the pure attacker-defender approach can be misleading for the hardening of a system against specific natural disasters. 
In this section, we propose a bi-level optimization model for identifying the vulnerabilities of a CI system under a specific hazard. The notations used in the model are given as follows:

\section{Indices, sets and parameters}

$i \quad$ Index used for buses (nodes)

$l \quad$ Index used for transmission lines

$V \quad$ Set of buses

$V_{G} \quad$ Set of generators

$V_{D} \quad$ Set of demand nodes

$L \quad$ Set of transmission lines

$\mathbb{T} \quad$ Set of discrete times of hazards

$\mathbb{U} \quad$ Uncertainty set of system component failures

$\mathbb{O}(\boldsymbol{u}) \quad$ Feasible set of system operation under a realization of an uncertainty scenario $\boldsymbol{u} \in \mathbb{U}$

$\tilde{R}(\boldsymbol{u}, \boldsymbol{o}) \quad$ System functionality loss under hazards

$T^{\max } \quad$ Maximal repair time of system components

$X_{l} \quad$ Reactance of transmission line $l$

$O(l) \quad$ Origin or sending node of line $l$

$R(l) \quad$ Destination or receiving node of line $l$

$G_{i}^{G} \quad$ Capacity of generator $i$

$F_{l}^{L} \quad$ Capacity of line $l$

$P_{i t}^{D} \quad$ Total demand at node $i$ at time $t$

$\theta^{\max } \quad$ Maximum allowable limit for $\theta_{i t}$ variables

$c_{i t}^{D} \quad$ Load shedding cost at node $i$ at time $t$

$\Gamma \quad$ Budget of failure uncertainty

$\Upsilon \quad$ Budget of recovery uncertainty

\section{Decision variables}

$y_{l t} \quad$ Binary variables indicating whether an overhead line $l$ is damaged to be offline $\left(y_{l t}=1\right)$ or not $\left(y_{l t}=0\right)$ at time $t$

$r_{l t} \quad$ Binary variables indicating whether an overhead line $l$ is restored to be online $\left(r_{l t}=1\right)$ or not $\left(r_{l t}=0\right)$ within time $t$

$x_{l t} \quad$ Binary variables indicating whether an overhead line $l$ is online (operational, $x_{l t}=1$ ) or not $\left(x_{l t}=0\right)$ at time $t$

$\theta_{i t} \quad$ Phase angle in node $i$ at time $t$

$f_{l t} \quad$ Power flow in line $l$ at time $t$

$g_{i t} \quad$ Power output at generator node $i \in V_{G}$ at time $t$

$\Delta P_{i t} \quad$ Load shedding in node $i \in V_{D}$

The proposed bi-level interdiction optimization is formulated as follows: 


$$
\max _{\boldsymbol{u} \in \mathbb{U}} \min _{\boldsymbol{o} \in \mathbb{O}(\boldsymbol{u})} \tilde{R}(\boldsymbol{u}, \boldsymbol{o})
$$

where

$$
\tilde{R}(\boldsymbol{u}, \boldsymbol{o})=\sum_{t \in \mathbb{T}} \sum_{i \in V_{D}} c_{i t}^{D} \Delta P_{i t}
$$

is the objective function representing the total system performance loss during a natural hazard event, where the variable $\boldsymbol{u}$ represents the time-dependent failure states of the transmission lines affected by the event; the variable $\boldsymbol{o}$ indicates the feasible system operation vector; $\mathbb{U}$ and $\mathbb{O}$ are the uncertainty set of transmission line failures and the feasible set of system operations, respectively. The objective function is calculated by the cumulative load shedding costs across all the demand nodes $V_{D}$ in the system and over the entire event duration $\mathbb{T}$. The first level problem in (8) aims to identify the most critical failure pattern of transmission lines $\boldsymbol{u}$ so that the system performance loss $\tilde{R}(\boldsymbol{u}, \boldsymbol{o})$ is maximized. The vector variable $\boldsymbol{u}$ includes information about which transmission lines are failed, and the associated failure and recovery times, which are modeled by the time-dependent indicator variables $\boldsymbol{x}, \boldsymbol{y}$ and $\boldsymbol{r}$, i.e., $\boldsymbol{u}=[\boldsymbol{x}, \boldsymbol{y}, \boldsymbol{r}]$. The second level problem is to minimize the system performance loss $\tilde{R}(\boldsymbol{u}, \boldsymbol{o})$ due to the physical damage of transmission lines in the second level via feasible system operations, i.e. redispatching of the power flows.

The uncertainty set $\mathbb{U}$ of component failures under a hazard is modeled as follows:

$$
\begin{aligned}
& \mathbb{U}=\left\{\boldsymbol{u} \mid \sum_{l \in L}\left(-\log _{2} p_{l t}\right) y_{l t} \leq \Gamma, \forall t \in \mathbb{T}\right. \\
& \sum_{t} y_{l t} \leq 1, \forall l \in L \\
& \sum_{l \in L}\left[-\log _{2} \bar{p}_{l, \text { repair }}(t \mid c a t g)\right] r_{l t} \leq \Upsilon, \forall t \in\left\{1, \ldots, T^{\text {max }}\right\} \\
& \sum_{t=1}^{T^{\max }} r_{l t} \leq 1, \forall l \in L \\
& x_{l t}+\sum_{t-\sum_{t} t \cdot r_{l t}}^{t} y_{l t}=1, l \in L, t \in \mathbb{T} \\
& \left.x_{l t}, y_{l t}, r_{l t} \in\{0,1\}, \forall l \in L, t \in \mathbb{T}\right\}
\end{aligned}
$$

where $\boldsymbol{u}=[\boldsymbol{x}, \boldsymbol{y}, \boldsymbol{r}]$ indicates the failure states of the overhead lines in the system over the whole time horizon of the hazard. Constraint (10) defines the uncertainty budget of system failure. Inspired by Shannon's information theory ("7th AIMMS-MOPTA Optimization Modeling Competition," 2015; Shannon, 2001), this definition relates the failure probabilities $\boldsymbol{p}$ of the system components and their binary damage variables $\boldsymbol{y}$ at each time period. The parameter $\Gamma$ represents the total uncertainty budget 
of system failure, and can be assigned by the analyst. As a matter of fact, the notion of uncertainty budget is widely used in robust optimization for allowing the decision maker to control the conservatism of the robust optimization formulation, which is inherently max-min (i.e., worst-case) (Ben-Tal, El Ghaoui, \& Nemirovski, 2009; Bertsimas, Brown, \& Caramanis, 2011). Generally, a large value of uncertainty budget represents a low level of risk that the decision maker is willing to take, i.e., the decision maker is conservative. By this approach, we provide the decision maker with a knob to adjust for her/his risk attitude. In the constraint (10), the failure probability $p_{l t}$ is calculated by Eq. (3). The constraint (10) states that the failure of a "reliable" line, i.e., having smaller failure probability $p_{l t}$, is more "surprising", i.e., takes up more failure uncertainty budget than the failure of a vulnerable line that has a larger failure probability $p_{l t}$. For instance, if the failure probability of a line $p_{l t}=0$, then the occurrence of its failure takes an infinite large failure uncertainty budget and $y_{l t}$ will be 0 , if $\Gamma$ is not infinite. Conversely, if the failure probability $p_{l t}=1$, then the occurrence of its failure takes zero budget, and $y_{l t}$ will be 1 in the optimization. Therefore, given a vector $\boldsymbol{p}$ of the failure probability of the system components, a large $\Gamma$ implies a large system failure budget and thus a large upper limit of the number of failed lines. In other words, by setting a larger $\Gamma$ the decision maker is willing to take a lower level of risk (i.e., assuming a larger quantity of "bad luck") and, thus, obtains a larger set of worst-case failed lines (that he/she presumably wants to protect). Constraint (11) states that an overhead line can only fail once during the horizon of a hazard. Similar to constraint (10), constraint (12) bounds the uncertainty budget $\Upsilon$ for the component recovery times. A large value of $\Upsilon$ represents a high degree of uncertainty with regard to the restoration times of the failed lines. In other words, the decision maker expects a large quantity of "bad luck" and allows the failed lines to be restored with more "surprising" periods of time, e.g., longer periods of time with smaller probabilities. In (12), $\bar{p}_{\text {l,repair }}(t \mid c a t g)$ represents the normalized probability that a failed line $l$ is recovered within time duration $t\left(t \leq T^{\text {max }}\right)$ under a specific category of storm, and is calculated as follows:

$$
\begin{gathered}
p_{l, \text { repair }}(t \mid c a t g)=p_{l, \text { repair }}(t \mid c a t g)-p_{l, \text { repair }}(t-1 \mid \text { catg }) \\
\bar{p}_{l, \text { repair }}(t \mid \text { catg })=\frac{p_{l, \text { repair }}(t \mid \text { catg })}{\max _{t \in\left\{1, \ldots, T_{\text {max }}\right\}} p_{l, \text { repair }}(t \mid \text { catg })}
\end{gathered}
$$

where $p_{l, \text { repair }}(t \mid c a t g)$ is obtained by Eq. (7). The normalized probability is used here to ensure that the problem is still feasible when the uncertainty budget $\Upsilon$ takes the value of 0 . Specifically, it is noted that $\bar{p}_{l, \text { repair }}(t \mid c a t g)$ always takes the value of 1 for the time period with the largest probability, i.e., for $t^{*}=\operatorname{argmax} p_{l, \text { repair }}(t \mid c a t g)$; then, the recovery duration of a failed line $l$ will be $t^{*}$ in the optimization for $\Upsilon=0$. In practice, the values assigned to the parameter $\Upsilon$ can, for instance, reflect the decision maker's own attitude towards restoration uncertainty; the connection between risk preference and the budget of uncertainty set has been studied in depth in (Bertsimas et al., 2011; Bertsimas \& Thiele, 2006). Constraint (13) indicates that a failed line is repaired only once during a hazard. Constraint (14) 
imposes that a line is either functional, i.e., $x_{l t}=1$, or failed and not being repaired, i.e., $\sum_{t-\sum_{t} t \cdot r_{l t}} y_{l t}=$ 1 , where $\sum_{t} t \cdot r_{l t}$ gives the repair time of the line. Constraint (15) imposes the integrity conditions for the variables $\boldsymbol{x}, \boldsymbol{y}$ and $\boldsymbol{r}$.

In the second level of (8), the feasible set of system operations under a realization of an uncertainty scenario $\boldsymbol{u} \in \mathbb{U}$ for power grids is formulated by the DC power flow (Y. Fang \& Sansavini, 2017; Ouyang \& Fang, 2017) as follows:

$$
\begin{gathered}
\mathbb{Q}(\boldsymbol{u})=\left\{\boldsymbol{o} \mid X_{l} f_{l t}-\left[\theta_{O(l) t}-\theta_{R(l) t}\right] \stackrel{\alpha_{l t}}{\leq} M_{1}\left(1-x_{l t}\right), \forall l, t\right. \\
X_{l} f_{l t}-\left[\theta_{O(l) t}-\theta_{R(l) t}\right] \stackrel{\beta_{l t}}{\geq}-M_{1}\left(1-x_{l t}\right), \forall l, t \\
P_{i t}^{D} \stackrel{\gamma_{i t}}{=} g_{i t}+\sum_{l \in L \mid R(l)=i} f_{l t}-\sum_{l \in L \mid O(l)=i} f_{l t}+\Delta P_{i t}, \forall i, t \\
0 \leq g_{i t} \stackrel{\delta_{i t}}{\leq} G_{i}^{G}, \forall i, t \\
0 \leq \Delta P_{i t} \stackrel{\zeta_{i t}}{\leq} P_{i t}^{D}, \forall i, t \\
-x_{l t} F_{l}^{L} \stackrel{\eta_{l t}}{\leq} f_{l t} \stackrel{\rho_{l t}}{\leq} x_{l t} F_{l}^{L}, \forall l, t \\
-\theta^{\max } \stackrel{\lambda_{i t}}{\leq} \theta_{i t} \stackrel{\mu_{i t}}{\leq} \theta^{\max }, \forall i, t \\
\left.\theta_{t}^{\text {ref }} \stackrel{\vartheta_{t}^{r e f}}{=} 0, \forall t\right\}
\end{gathered}
$$

where constraints (18)-(19) represent the power flow for every line, where $M$ is a sufficiently large positive constraint (i.e., $M \geq 2 \theta^{\max }$ ). Constraint (20) enforces the power balance for each bus. Constraint (21) limits the capacities of generation units. Also, constraint (22) bounds the maximum value of unserved electricity demand for each bus. Constraint (23) sets the limits of power flow on each lines. Finally, constraint (24) bounds phase angles and constraint (25) sets the phase angle of the reference bus to zero. The feasible operation of the system is represented by continuous variables $g_{i t}, f_{l t}, \Delta P_{i t}, \theta_{i t}$ and $\theta_{t}^{r e f}$. In (18)-(25), we denote the dual variables associated with each constraints that will be used in Section (4).

\section{SOLUTION METHOD}

The proposed max-min formulation (8)-(25) of Section 3 configures a bi-level mix-integer linear programming (MILP) problem. The solution method presented in this section casts the problem into an equivalent one-level MILP. For a given upper level decision vector $\boldsymbol{u}$, the lower-level system operation problem

$$
\min _{\boldsymbol{o} \in \mathbb{O}(\boldsymbol{u})} \tilde{R}(\boldsymbol{o}) \text { where } \mathbb{O}(\boldsymbol{u}) \text { is subject to }(18)-(25)
$$


is a pure linear programming (LP) problem, hereinafter referred to as the primal of the lower-level problem (or simply primal problem).

Proposition 1: For every given upper-level decision vector $\boldsymbol{u}=[\boldsymbol{x}, \boldsymbol{y}, \boldsymbol{r}]$ and for bounded $c_{i t}^{D}$, the primal of the lower-level problem (26) has a finite optimum.

Proof: It suffices to show that the problem (26) is neither unbounded nor infeasible for any given upper level decision vector $[\boldsymbol{x}, \boldsymbol{y}, \boldsymbol{r}]$. Note that all decision variables in (26), i.e., $\boldsymbol{g}, \boldsymbol{f}, \Delta \boldsymbol{P}, \boldsymbol{\theta}$ and $\boldsymbol{\theta}^{\boldsymbol{r e f}}$, have finite lower and upper bounds, which is consistent with the physical requirements of power system operation. Moreover, $\left[\boldsymbol{g}, \boldsymbol{f}, \boldsymbol{\theta}, \boldsymbol{\theta}^{\text {ref }}\right]=0$ and $\Delta \boldsymbol{P}=\boldsymbol{P}^{\boldsymbol{D}}$ is always a feasible solution to the problem, for any values of $[\boldsymbol{x}, \boldsymbol{y}, \boldsymbol{r}]$.

The dual problem of the lower-level primal problem is given as follows:

$$
\begin{aligned}
\max _{d \in \mathbb{D}(\boldsymbol{u})} \sum_{t \in \mathbb{T}}\left\{\sum_{l \in L}\left[F_{l}^{L} x_{l t}\left(\rho_{l t}-\eta_{l t}\right)+M_{1}\left(1-x_{l t}\right)\left(\alpha_{l t}-\beta_{l t}\right)\right]\right. \\
\left.+\sum_{i \in V}\left[P_{i t}^{D}\left(\gamma_{i t}+\zeta_{i t}\right)+\theta^{\max }\left(\mu_{i t}-\lambda_{i t}\right)+G_{i}^{G} \delta_{i t}\right]\right\}
\end{aligned}
$$

Subject to

$$
\begin{aligned}
& \mathbb{D}(\boldsymbol{u})=\left\{\boldsymbol{d} \mid \gamma_{i t}+\delta_{i t} \stackrel{g_{i t}}{\leq} 0, \forall i \in V, t \in \mathbb{T}\right. \\
& \gamma_{i t}+\zeta_{i t} \stackrel{\Delta P_{i t}}{\leq} c_{i t}^{D}, \forall i \in V, t \in \mathbb{T} \\
& X_{l} \alpha_{l t}+X_{l} \beta_{l t}+\gamma_{R(l) t}-\gamma_{o(l) t}+\rho_{l t}+\eta_{l t} \stackrel{f_{l t}}{=} 0, \forall l \in L, t \in \mathbb{T} \\
& \sum_{(l \in L \mid R(l)=i)}\left(\alpha_{l t}+\beta_{l t}\right)-\sum_{(l \in L \mid O(l)=i)}\left(\alpha_{l t}+\beta_{l t}\right)+\lambda_{i t}+\mu_{i t} \stackrel{\theta_{i t}}{=} 0, \forall i \in V \backslash r e f, t \in \mathbb{T} \\
& \sum_{(l \in L \mid R(l)=i)}\left(\alpha_{l t}+\beta_{l t}\right)-\sum_{(l \in L \mid O(l)=i)}\left(\alpha_{l t}+\beta_{l t}\right)+\lambda_{i t}+\mu_{i t}+\vartheta_{t}^{i} \stackrel{\theta_{t}^{i}}{=} 0 \text {, for } i=r e f, t \in \mathbb{T} \\
& \alpha_{l t} \leq 0, \beta_{l t} \geq 0, \eta_{l t} \geq 0, \rho_{l t} \leq 0, \forall l \in L, t \in \mathbb{T} \\
& \left.\delta_{i t} \leq 0, \zeta_{i t} \leq 0, \lambda_{i t} \geq 0, \mu_{i t} \leq 0, \forall i \in V, t \in \mathbb{T}\right\}
\end{aligned}
$$

where $\boldsymbol{d}$ represents the vector of dual variables and its feasible space $\mathbb{D}(\boldsymbol{u})$ is given by (28)-(34), in which the primal variables that each dual constraints correspond to are defined.

Regarding the optimality of the lower-level primal problem (26) and its dual problem (27)-(34), the following property exists:

Proposition 2: For a given upper-level decision vector $\boldsymbol{u}=[\boldsymbol{x}, \boldsymbol{y}, \boldsymbol{r}]$, suppose $\left[\boldsymbol{g}, \boldsymbol{f}, \Delta \boldsymbol{P}, \boldsymbol{\theta}, \boldsymbol{\theta}^{\boldsymbol{r e f}}\right]$ is a feasible solution to the lower-level primal problem (26) and $\left[\boldsymbol{\alpha}, \boldsymbol{\beta}, \boldsymbol{\gamma}, \boldsymbol{\eta}, \boldsymbol{\rho}, \boldsymbol{\delta}, \boldsymbol{\zeta}, \boldsymbol{\lambda}, \boldsymbol{\mu}, \boldsymbol{\vartheta}^{\text {ref }}\right]$ is a feasible solution to problem (27)-(34). Then, $\left[\boldsymbol{g}, \boldsymbol{f}, \Delta \boldsymbol{P}, \boldsymbol{\theta}, \boldsymbol{\theta}^{\text {ref }}\right]$ is an optimal solution to (26), and $\left[\boldsymbol{\alpha}, \boldsymbol{\beta}, \boldsymbol{\gamma}, \boldsymbol{\eta}, \boldsymbol{\rho}, \boldsymbol{\delta}, \zeta, \boldsymbol{\lambda}, \boldsymbol{\mu}, \boldsymbol{\vartheta}^{\text {ref }}\right]$ is an optimal solution to (27)-(34), if and only if 


$$
\begin{aligned}
\sum_{t \in \mathbb{T}} \sum_{i \in V_{D}} c_{i t}^{D} \Delta P_{i t} & =\sum_{t \in \mathbb{T}}\left\{\sum_{l \in L}\left[F_{l}^{L} x_{l t}\left(\rho_{l t}-\eta_{l t}\right)+M_{1}\left(1-x_{l t}\right)\left(\alpha_{l t}-\beta_{l t}\right)\right]\right. \\
& \left.+\sum_{i \in V}\left[P_{i t}^{D}\left(\gamma_{i t}+\zeta_{i t}\right)+\theta^{\max }\left(\mu_{i t}-\lambda_{i t}\right)+G_{i}^{G} \delta_{i t}\right]\right\} .
\end{aligned}
$$

Proof: A proof can be easily completed by evoking Proposition 1 together with the LP strong duality theorem which is a necessary condition of Proposition 2 and a direct consequence of the weak duality theorem which is a sufficient condition of Proposition 2.

Therefore, based on Proposition 2, the bi-level problem (8)-(25) can be transformed into the following single-level maximization problem by considering the dual of the inner-level problem:

$$
\begin{aligned}
\max _{\boldsymbol{u} \in \mathbb{U}, \boldsymbol{d} \in \mathbb{D}(\boldsymbol{u})} \sum_{t \in \mathbb{T}}\left\{\sum_{l \in L}\left[F_{l}^{L} x_{l t}\left(\rho_{l t}-\eta_{l t}\right)+M_{1}\left(1-x_{l t}\right)\left(\alpha_{l t}-\beta_{l t}\right)\right]\right. \\
\left.+\sum_{i \in V}\left[P_{i t}^{D}\left(\gamma_{i t}+\zeta_{i t}\right)+\theta^{\max }\left(\mu_{i t}-\lambda_{i t}\right)+G_{i}^{G} \delta_{i t}\right]\right\}
\end{aligned}
$$

subject to

$$
\text { (10)-(15) and (28)-(34) }
$$

The bilinear terms in the objective function of (36), i.e., $x_{l t}\left(\rho_{l t}-\eta_{l t}\right)$ and $\left(1-x_{l t}\right)\left(\alpha_{l t}-\beta_{l t}\right)$, can be linearized using linearization schemes that have been previously reported in the MILP literature (e.g., see Y. Fang and Sansavini (2017) and Ouyang and Fang (2017)). Specifically, we replace $x_{l t} \rho_{l t}$ and $x_{l t} \eta_{l t}$ with $\tilde{\rho}_{l t}$ and $\tilde{\eta}_{l t}$, respectively, and introduce the following four additional constraints:

$$
\begin{gathered}
\rho_{l t} \leq \tilde{\rho}_{l t} \leq 0, \forall l, t \\
-M_{2} x_{l t} \leq \tilde{\rho}_{l t} \leq \rho_{l t}+M_{2}\left(1-x_{l t}\right), \forall l, t \\
0 \leq \tilde{\eta}_{l t} \leq \eta_{l t}, \forall l, t \\
\eta_{l t}-M_{2}\left(1-x_{l t}\right) \leq \tilde{\eta}_{l t} \leq M_{2} x_{l t}, \forall l, t
\end{gathered}
$$

where $M_{2}$ is a sufficiently large number. Likewise, we replace the bilinear terms $\left(1-x_{l t}\right) \alpha_{l t}$ and $\left(1-x_{l t}\right) \beta_{l t}$ with $\tilde{\alpha}_{l t}$ and $\tilde{\beta}_{l t}$, respectively, and introduce four additional constraints:

$$
\begin{gathered}
\alpha_{l t} \leq \tilde{\alpha}_{l t} \leq 0, \forall l, t \\
-M_{2}\left(1-x_{l t}\right) \leq \tilde{\alpha}_{l t} \leq \alpha_{l t}+M_{2} x_{l t}, \forall l, t \\
0 \leq \tilde{\beta}_{l t} \leq \beta_{l t}, \forall l, t \\
\beta_{l t}-M_{2} x_{l t} \leq \tilde{\beta}_{l t} \leq M_{2}\left(1-x_{l t}\right), \forall l, t
\end{gathered}
$$

Consequently, the single level problem (36) is recast into an equivalent MILP, summarized as follows: 


$$
\begin{aligned}
\max _{\boldsymbol{u} \in \mathbb{U}, \boldsymbol{d} \in \mathbb{\mathbb { D }}(\boldsymbol{u})} \sum_{t \in \mathbb{T}}\left\{\sum_{l \in L}\left[F_{l}^{L}\left(\tilde{\rho}_{l t}-\tilde{\eta}_{l t}\right)+M_{1}\left(\tilde{\alpha}_{l t}-\tilde{\beta}_{l t}\right)\right]\right. \\
\left.+\sum_{i \in V}\left[P_{i t}^{D}\left(\gamma_{i t}+\zeta_{i t}\right)+\theta^{\max }\left(\mu_{i t}-\lambda_{i t}\right)+G_{i}^{G} \delta_{i t}\right]\right\}
\end{aligned}
$$

subject to

$$
\text { (10)-(15), (28)-(34) and (37)-(44). }
$$

This resulting MILP problem is solved by global optimization solvers such as CPLEX (IBM, 2015).

\section{CASE STUDY}

\subsection{Data Set}

To illustrate the proposed vulnerability analysis approach, the IEEE 24-bus one area reliability test system (named as RTS24) is considered (Grigg et al., 1999). To embed the system into a specific territory, the line lengths and geographical locations are chosen following Mohanpurkar, Sogbi, and Suryanarayanan (2015). Bus 7 of the test system is taken as a reference node and is located near Xiamen (24.5 N, 118.0E), a coastal city in China. The system is georeferenced by projecting it onto a $400 \times 400 \mathrm{~km}^{2}$ study area located in the South of China, as illustrated in Fig. 3. There are 24 nodes (buses) and 38 transmission lines (circuits). The georeferenced coordinates of all nodes are given in Table II.

In the present study, we use analytical fragility curves for power towers and transmission lines (between two supporting towers) adapted from Panteli, Pickering, Wilkinson, Dawson, and Mancarella

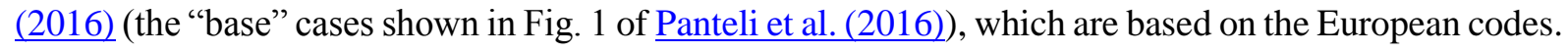
The parameters setting of $w_{\text {critical }}$ and $w_{\text {collapse }}$ are the same as those in Panteli et al. (2016). The fallen tree-induced failure probability of conductors is not considered for simplicity. However, this can be easily incorporated when the related data are available. As for the level of damage for towers and transmission lines, binary states are considered, i.e., a component is either completely out of service or fully operational, and the likelihood of complete failure is modeled using the analytical fragility curves. 


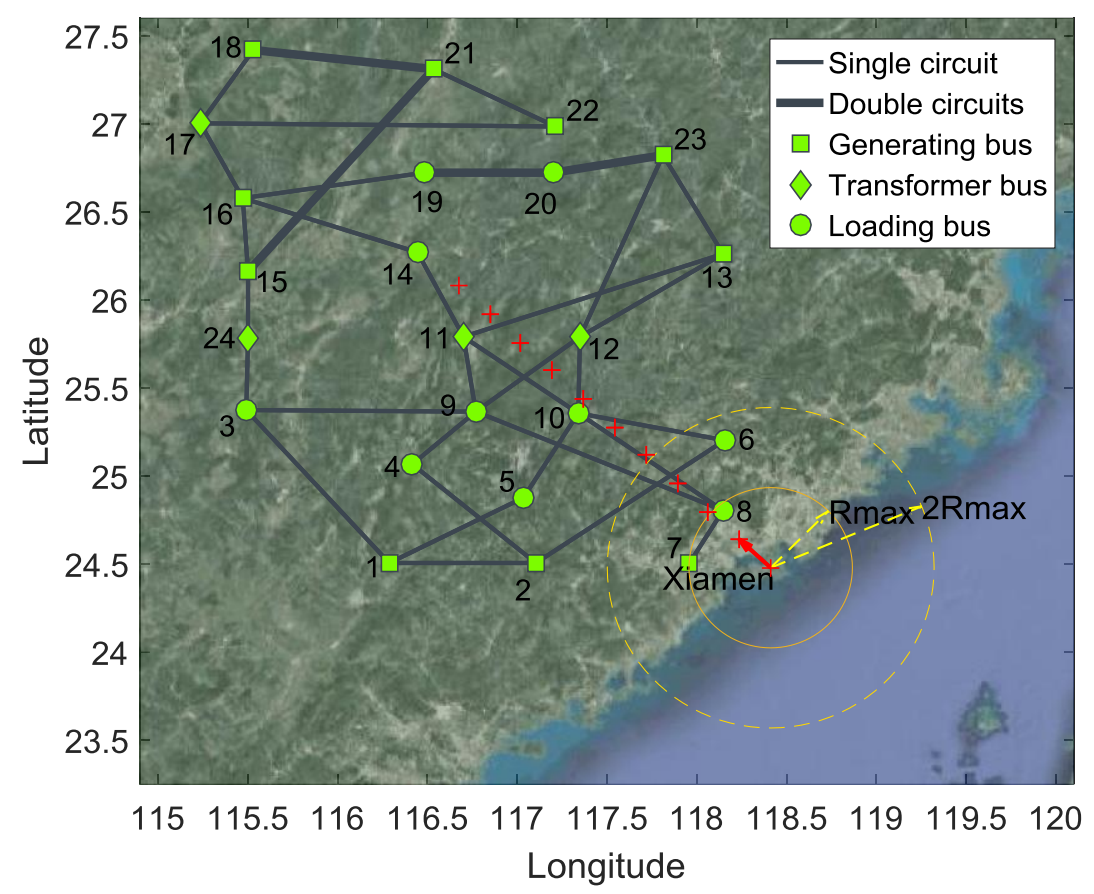

Fig. 3. The georeferenced RTS24 test system and one realization of a Catg-1 storm track.

Table II. Geographical coordinates of power system buses

\begin{tabular}{crr|rrr|rrr}
\hline \hline Node ID & Longitude & Latitude & Node ID & Longitude & Latitude & Node ID & Longitude & Latitude \\
\hline 1 & $116.288 \mathrm{E}$ & $24.507 \mathrm{~N}$ & 9 & $116.770 \mathrm{E}$ & $25.366 \mathrm{~N}$ & 17 & $115.236 \mathrm{E}$ & $27.003 \mathrm{~N}$ \\
2 & $117.104 \mathrm{E}$ & $24.507 \mathrm{~N}$ & 10 & $117.340 \mathrm{E}$ & $25.357 \mathrm{~N}$ & 18 & $115.531 \mathrm{E}$ & $27.420 \mathrm{~N}$ \\
3 & $115.492 \mathrm{E}$ & $25.375 \mathrm{~N}$ & 11 & $116.701 \mathrm{E}$ & $25.791 \mathrm{~N}$ & 19 & $116.485 \mathrm{E}$ & $26.723 \mathrm{~N}$ \\
4 & $116.416 \mathrm{E}$ & $25.067 \mathrm{~N}$ & 12 & $117.350 \mathrm{E}$ & $25.791 \mathrm{~N}$ & 20 & $117.203 \mathrm{E}$ & $26.723 \mathrm{~N}$ \\
5 & $117.035 \mathrm{E}$ & $24.878 \mathrm{~N}$ & 13 & $118.147 \mathrm{E}$ & $26.262 \mathrm{~N}$ & 21 & $116.534 \mathrm{E}$ & $27.311 \mathrm{~N}$ \\
6 & $118.156 \mathrm{E}$ & $25.203 \mathrm{~N}$ & 14 & $116.445 \mathrm{E}$ & $26.271 \mathrm{~N}$ & 22 & $117.212 \mathrm{E}$ & $26.985 \mathrm{~N}$ \\
7 & $117.960 \mathrm{E}$ & $24.507 \mathrm{~N}$ & 15 & $115.501 \mathrm{E}$ & $26.162 \mathrm{~N}$ & 23 & $117.812 \mathrm{E}$ & $26.823 \mathrm{~N}$ \\
8 & $118.147 \mathrm{E}$ & $24.805 \mathrm{~N}$ & 16 & $115.472 \mathrm{E}$ & $26.578 \mathrm{~N}$ & 24 & $115.501 \mathrm{E}$ & $25.782 \mathrm{~N}$ \\
\hline \hline
\end{tabular}

\subsection{Wind Storm Simulation}

For illustration purposes, the storms hit at the location with latitude $24.50 \mathrm{~N}$ and longitude $118.30 \mathrm{E}$ (near Xiamen). The storms are assumed to be moving with a translational speed of $25 \mathrm{~km} / \mathrm{h}$ and traveling towards northwest $\left(135^{\circ}\right)$ (Dorst, 2017). Fig. 3 illustrates one realization of the forecasted track of the storms. The red plus signs represent the locations of the storm eye at different times, with one hour time steps. The yellow circle indicates the boundary of the maximum winds for the traveling storms at the landfall point. The area between the yellow circle $R_{\max }$ and the dashed yellow circle $2 R_{\max }$ experiences around $82.5 \%$ of the maximum wind speed.

In order to assess the wind storm impact on the different elements of the RTS24 system, its dynamic wind field is modelled through Eq. (1), from which we can calculate the time-varying wind speeds at 
each location within the power system. We consider three moderate-to-extreme wind storms, i.e. category-1, category-2 and category-3 with their maximal wind speed $V_{m}=38 \mathrm{~m} / \mathrm{s}, 46 \mathrm{~m} / \mathrm{s}$ and $54 \mathrm{~m} / \mathrm{s}$, respectively. Fig. 4 shows the surface wind gust speed variations at node 7 within the test system as the storms of the studied three categories travel along their tracks.

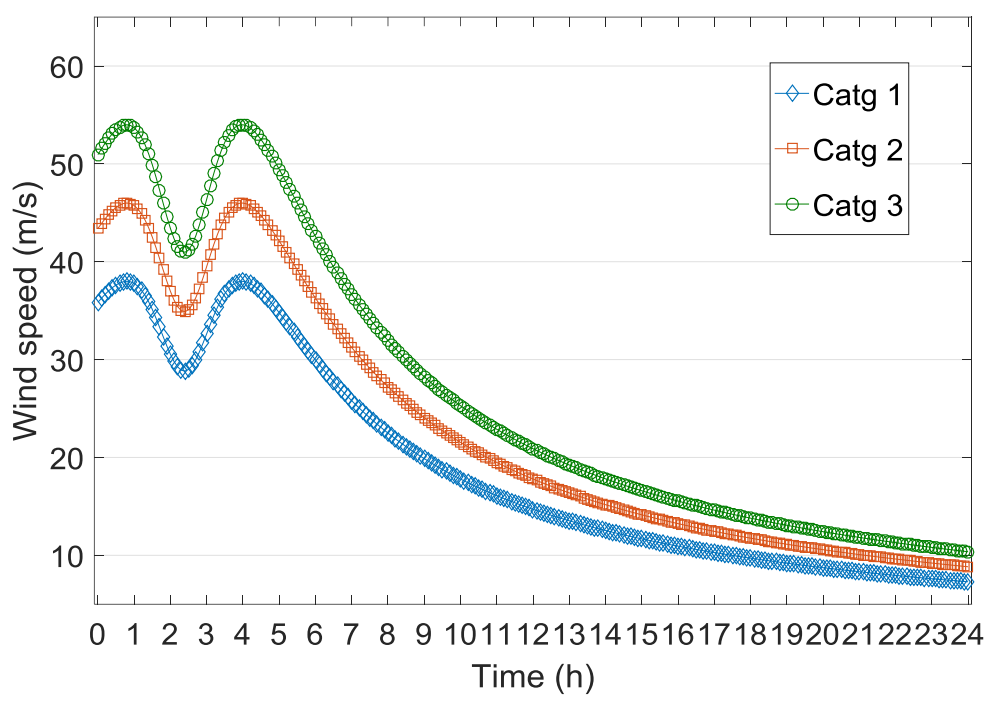

Fig. 4. Hourly wind gust profiles at node no.7 under different categories of wind storms.

For the recovery time of the failed transmission lines, we assume $M T T R_{l}=10 \mathrm{hrs}$ and $\sigma=1$ for overhead lines (Espinoza et al., 2016; Zapata et al., 2008). As storm intensity increases, the repair crews need more time to approach the affected area and restore the damaged components, therefore, the restoration stress is assumed to be $R S_{\text {catg }}=1,2$, and 3 for category-1, category- 2 and category- 3 wind storms, respectively. Due to the lack of historical restoration data for the IEEE test system, the distribution parameters are assumed based on the related literature. The development of probabilistic restoration models for power system components based on historical data is of practical interest. Yet, regardless of the developed probabilistic model, the probability values of the restoration times of the failed components can be incorporated into the proposed optimization framework via the constraints (10)-(15). This entails no additional burden to the proposed vulnerability analysis framework, whose development is the focus of the present work.

\subsection{Results}

Based on the above wind storm simulation and the geographic and structural fragility data of the test system, the failure probability of transmission lines can be calculated using Eqs. (2)-(4). The recovery probability of failed lines are calculated by Eq. (7), where the data for the MTTR and RS parameters of the transmission lines are based on Ouyang and Dueñas-Osorio (2014) and Espinoza et al. (2016). The proposed optimization problem for component criticality analysis is converted into its equivalent MILP problem following Section 4. The resulting MILP problem is implemented and solved in the IBM 
CPLEX 12.6 optimization studio (IBM, 2015). All calculations are performed on a laptop with 2.6-GHz CPU and 8GB RAM.

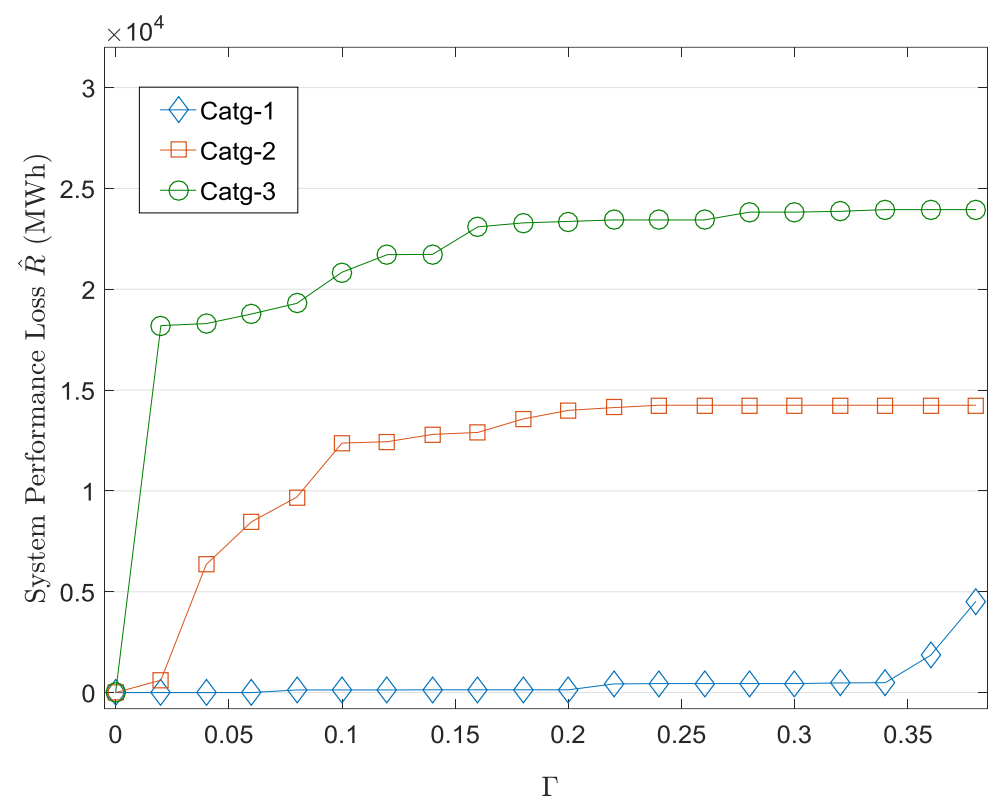

Fig. 5. Optimized worst case load shedding (system performance loss $\tilde{R}$ ) under different failure uncertainty $\Gamma$ at $\Upsilon=0.1$.

Fig. 5 shows the optimized worst-case system performance loss $\tilde{R}$ in terms of load shedding under different budgets of failure uncertainty $\Gamma$ when $\Upsilon=0.1$, for the three categories of storms. It can be seen that a severe storm generally results in large system load shedding for each $\Gamma$ because the large wind speeds increase the failure probabilities of the system components. Fig. 6 shows the time-dependent failure probabilities of all the 38 transmission lines in the RTS24 system under a) category-1, b) category-2 and c) category-3 storms, respectively. The failure probability of transmission lines is not likely to exceed 0.8 for the category-1 storm as shown in Fig. 6(a), whereas the number of lines whose failure probabilities exceed 0.9 increases largely for the category-2 storm in Fig. 6(b) and especially for the category-3 storm in Fig. 6(c). 

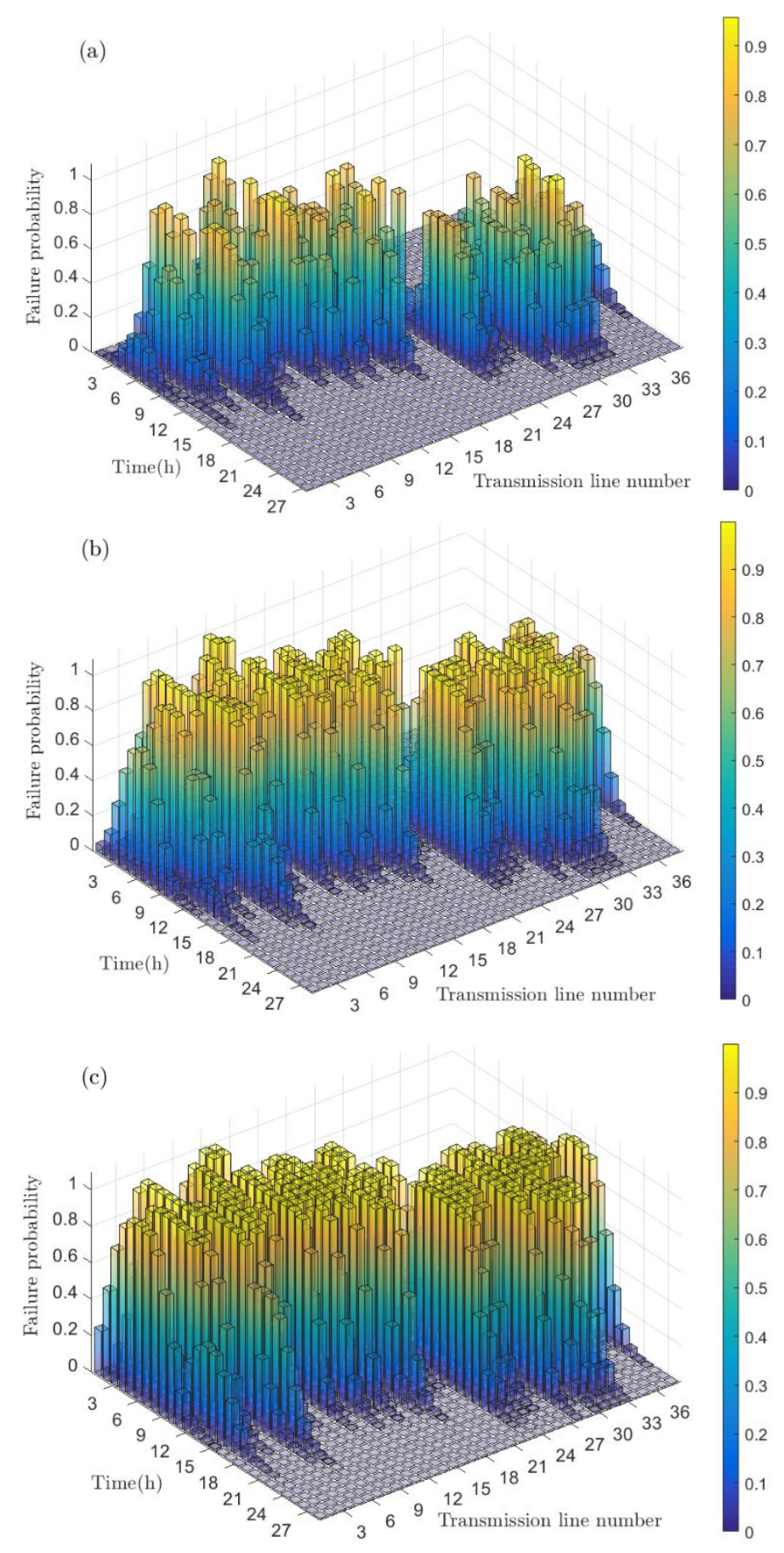

Fig. 6. Time-dependent failure probabilities of the transmission lines in the test system under (a) category-1, (b) category-2 and (c) category-3 storms.

Furthermore, Fig. 5 shows that the performance loss increases as the failure uncertainty budget $\Gamma$ increases, for all the three storm severities. This is because a bigger value of $\Gamma$ represents a larger upper limit of the number of failed lines, and indicates that the decision maker is more tolerant to loss of system performance. Therefore, the decision maker obtains a larger set of worst-case failed line by setting a large $\Gamma$. This can be further shown in Table III where the set of worst-case failed lines (Column 2) attained by the optimization for different values of $\Gamma$ and their corresponding load shedding (Column 3) for the category- 1 storm are listed. For instance, when $\Gamma=0.14$ the optimized worst-case failed lines 
are Line 8-9, Line 11-13 and Line 17-22, resulting in a total amount of 136.6MWh load shedding. It is important that the different sets of worst-case failed lines offer decision makers the flexibility to choose the appropriate critical lines to be protected. Moreover, Table III shows that the optimum set of the worst-case failed lines at small values of $\Gamma$ is not necessarily a subset of the lines to be failed at large values of $\Gamma$. For example, Line 8-9 is failed in the optimized worst-case when $\Gamma=0.14$ (row 3 ) and 0.22 (row 4), but it is replaced by Line 2-6 and Line 3-9 when $\Gamma=0.23$ (row 5) and 0.32 (row 6); Line 1722 is identified critical in rows 2-7 but not in rows 8-9.

Table III. Optimized worst-case failed lines and load shedding (LS) for the Catg-1 storm at $\Upsilon=0.1$

\begin{tabular}{cll}
\hline \hline$\Gamma$ & \multicolumn{1}{c}{ Worst-case failed lines } & LS (MWh) \\
\hline 0.07 & $17-22$ & 130.1 \\
0.14 & $8-9,11-13,17-22$ & 136.6 \\
0.22 & $8-9,11-13,12-23,17-22$ & 427.2 \\
0.23 & $2-6,3-9,11-13,12-23,17-22$ & 446.0 \\
0.32 & $2-6,3-9,11-13,12-23,17-22$ & 478.4 \\
0.35 & $2-6,3-9,9-12,11-13,12-23,17-22$ & 590.3 \\
0.36 & $2-6,3-9,8-9,9-12,11-13,12-23,16-19,17-22$ & 1849.2 \\
0.37 & $2-6,3-9,8-9,9-12,11-13,12-13,12-23,14-16,16-19$ & 3978.7 \\
0.38 & $2-6,3-9,6-10,8-9,9-12,11-13,12-13,12-23,14-16,16-19$ & 4522.7 \\
\hline \hline
\end{tabular}

Unlike the static network interdiction problems (Wood, 2011) in which the failure of a set of lines results in a fixed system performance loss, in the proposed model the failures of lines are time-dependent due to the spatiotemporal dynamic of natural hazards. Thus, the failures of a same set of lines may lead to different amounts of load shedding if their failures times are different, as we can observe in the rows 5 and 6 of Table III. For better illustration, Fig. 7(a) and Fig. 7(b) show the detailed failure and recovery times of each failed lines for the cases of $\Gamma=0.23$ (row 5 in Table III) and $\Gamma=0.32$ (row 6 in Table III), respectively. The failure and restoration times of Line 3-9 and Line 17-22 differ in the two cases, i.e., the former fails at the beginning of period $t=10$ and is repaired at the end of $t=17$ for $\Gamma=0.23$, whereas it fails at $t=8$ and is repaired at $t=15$ for $\Gamma=0.32$; the non-functional period of the latter starts from $t=18$ to $t=25$ for $\Gamma=0.23$, but it starts from $t=17$ to $t=24$ for $\Gamma=0.32$. This leads to a difference in the system load shedding of up to $32.4 \mathrm{MWh}$ for the two cases. In practice, the possible failure times of lines might be used to inform the decision maker of his/her preparation time allowance. Thus, the time allowance obtained by setting a larger value of failure uncertainty budget $\Gamma$, i.e., by allowing more "surprising" event to happen, is more robust for the preparation practice. 


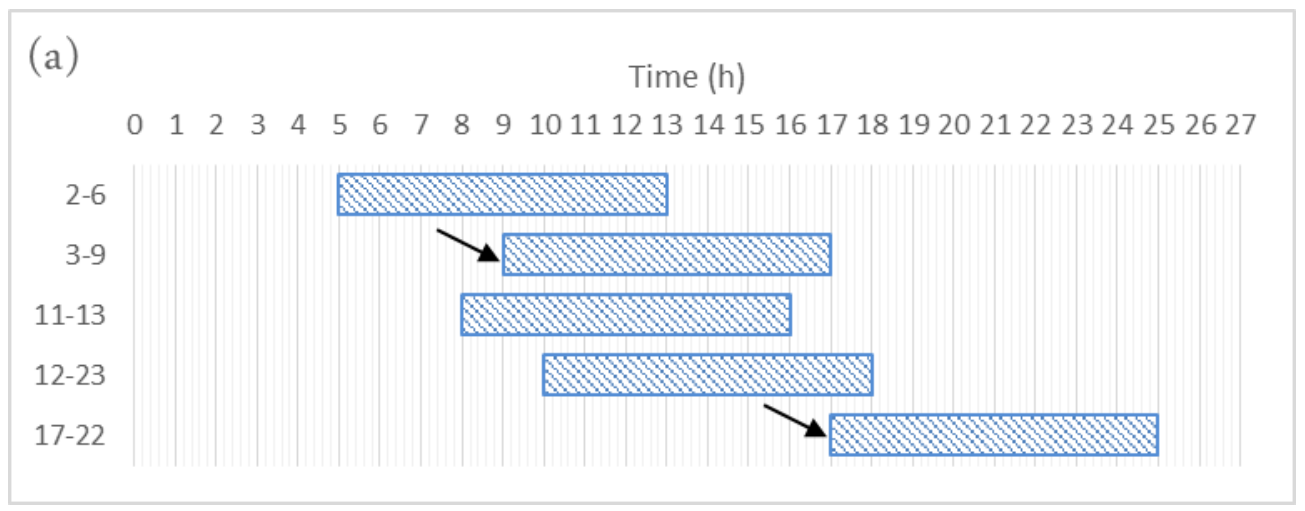

(b)

Time (h)

$0 \begin{array}{lllllllllllll}0 & 2 & 3 & 4 & 5 & 6 & 7 & 8 & 9 & 1011 & 12131415161718192021222324252627\end{array}$

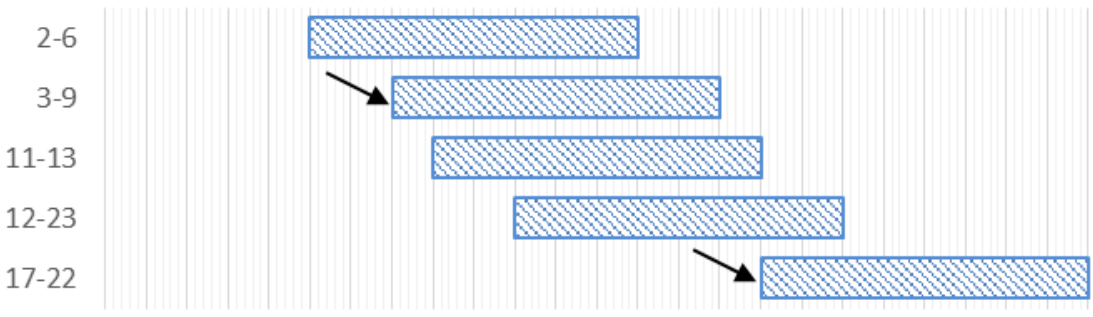

Fig. 7. Failure and restoration times of the worst-case failed lines for the Catg-1 storm at $\Upsilon=0.1$ for (a) $\Gamma=$ 0.23 and (b) $\Gamma=0.32$

Table IV and Table V report the optimized worst-case failed lines and load shedding for the category2 and category-3 storms, respectively. For larger storms, smaller values of failure uncertainty budget $\Gamma$ should be taken to obtain the same number of worst-case failed lines to that of smaller storms. This is due to the fact that more severe storms cause higher failure probabilities of overhead lines, as shown in Fig. 6; thus, less uncertainty budget is taken up by individual lines. Furthermore, the comparison of the optimized results in Table III, Table IV and Table V for the three categories of storms shows that stronger storms generally result in larger amounts of load shedding when a same set of lines are failed. For example, the performance loss for the category-1, category- 2 and category- 3 storms, is $478.4 \mathrm{MWh}$, 614.4MWh and 809.3MWh, respectively, when the same set of lines, i.e., Lines 2-6, 3-9, 11-13, 12-23 and 17-22, fails (rows 6 in Table III, Table IV and Table V). This is not unexpected since it often takes longer times to be restored for lines damaged in stronger storms, as represented in Eq. (7). Furthermore, Table III, Table IV and Table V show that very similar results regarding the worst-case failed lines are obtained for the three storms. This is probably because the three storms are assumed to have exactly the same landfall point and traveling direction, thus being characterized by a similar wind field though with different degrees of strength.

Discovering the worst-case failed system components and load shedding for a specific upcoming storm has important practical considerations for forecasting system damage and advising defensive actions. We see from Table III, Table IV and Table V that large values of the failure uncertainty budget $\Gamma$ correspond to large sets of worst-case failed transmission lines and usually large monetary costs for 
their protection. Based on these Tables, the decision makers can, therefore, select the sets of most critical lines to be hardened that are in line with their investment budget.

Table IV. Optimized worst-case failed lines and load shedding for the Catg- 2 storm at $\Upsilon=0.1$

\begin{tabular}{cll}
\hline \hline$\Gamma$ & \multicolumn{1}{c}{ Worst-case failed lines } & LS (MWh) \\
\hline 0.001 & $17-22$ & 162.6 \\
0.002 & $11-13,17-22$ & 172.4 \\
0.005 & $11-13,12-23,17-22$ & 533.2 \\
0.010 & $2-6,3-9,11-13,12-23,17-22$ & 586.7 \\
0.020 & $2-6,3-9,11-13,12-23,17-22$ & 614.4 \\
0.021 & $2-6,3-9,9-12,11-13,12-23,16-19,17-22$ & 2240.6 \\
0.022 & $2-6,3-9,9-12,11-13,12-13,12-23,14-16,16-19$ & 5247.5 \\
0.023 & $2-6,3-9,6-10,8-9,11-13,12-13,12-23,14-16,16-19,17-22$ & 5848.7 \\
0.024 & $2-6,3-9,6-10,8-9,8-10,9-12,11-13,12-13,12-23,14-16,16-19$ & 6209.6 \\
\hline \hline
\end{tabular}

Table V. Optimized worst-case failed lines and load shedding for the Catg-3 storm at $\Upsilon=0.1$

\begin{tabular}{lll}
\hline \hline$\Gamma\left(10^{-4}\right)$ & \multicolumn{1}{c}{ Worst-case failed lines } & LS (MWh) \\
\hline 0.10 & $8-9,11-13$ & 0 \\
0.30 & $11-13,12-23$ & 618.9 \\
0.35 & $2-6,3-9,11-13,12-23$ & 708.3 \\
0.50 & $2-6,3-9,11-13,12-23,17-22$ & 791.4 \\
1.00 & $2-6,3-9,11-13,12-23,17-22$ & 809.3 \\
4.50 & $2-6,3-9,9-12,11-13,12-13,12-23,16-19$ & 3824.1 \\
4.60 & $2-6,3-9,8-9,9-12,10-11,11-13,12-13,12-23,16-19$ & 3842.0 \\
4.80 & $2-6,3-9,6-10,11-13,12-13,12-23,14-16,16-19$ & 6960.8 \\
4.90 & $2-6,3-9,6-10,8-9,9-12,10-11,11-13,12-13,12-23,14-16,15-21,16-19$ & 6974.6 \\
\hline \hline
\end{tabular}

Finally, we consider multiple landfall points and directions of different category-1 storms and find the solutions for each storm. We consider 5 different storm scenarios around the test system as shown in Fig. 8. The optimized solutions regarding the worst-case failed lines and the corresponding load shedding are shown in Table VI. For each scenario, three different values of failure uncertainty budget are considered. Table VI shows that the optimized critical lines are quite different in the storms with various trajectories, although there is some overlapping of the worst-case failed lines, e.g., Line 17-22 in scenarios 1,2 and 5. No single line is identified critical in all the five storms. These results indicate that the landfall locations and directions of storms do affect the uncertain set of system component failures. This is due to the fact that the considered test system spans a quite large scale of geographical space and the impact of a storm on the system components is site-dependent. This contradicts the assumptions made in previous studies where the wind speed at the central point of a system is applied uniformly to all components of the system (Salman et al., 2015). 


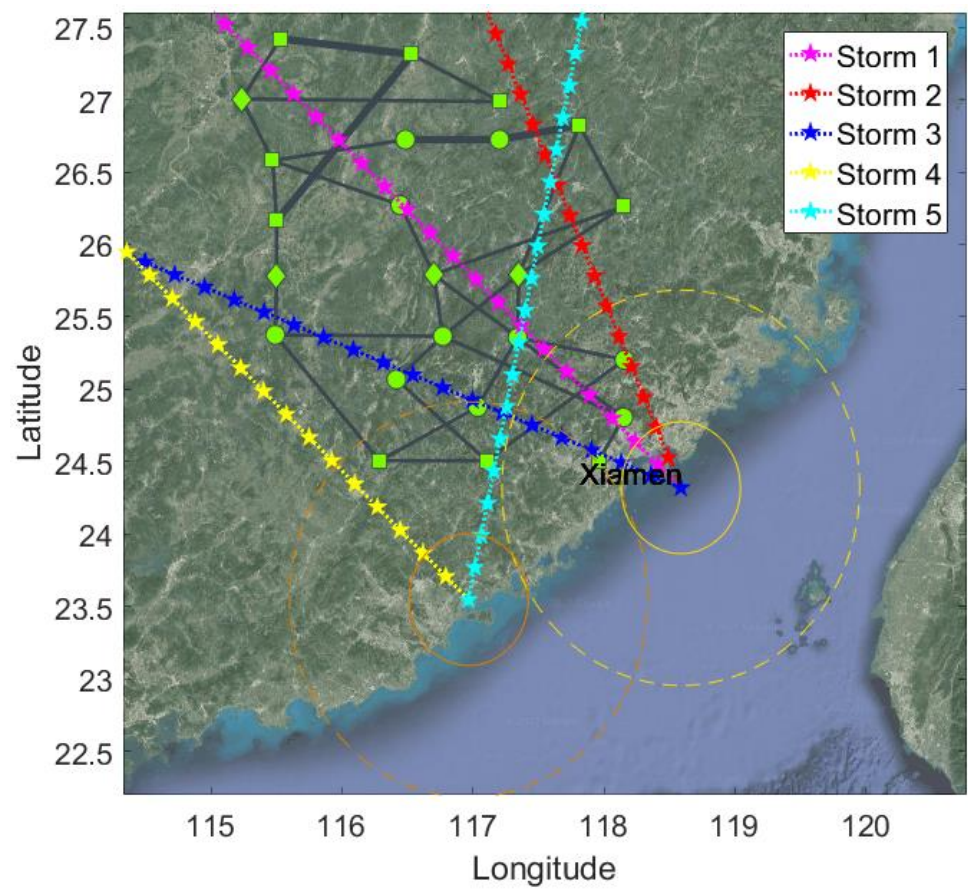

Fig. 8. Tracks of different wind storms with different landfall points and traveling directions

Table VI. Optimized worst-case failed lines and load shedding for different scenarios of category-1 storms where "NO" means no failure lines

\begin{tabular}{|c|c|c|c|c|}
\hline Scenarios & $\Gamma$ & Worst-case failed lines & LS (MWh) & Computation time (s) \\
\hline \multirow{3}{*}{ Storm 1} & 0.08 & $17-22$ & 130.1 & 0.4 \\
\hline & 0.23 & $2-6,3-9,11-13,12-23,17-22$ & 446.0 & 172 \\
\hline & 0.38 & $\begin{array}{l}2-6,3-9,6-10,8-9,9-12,11-13,12-13,12-23,14- \\
16,16-19\end{array}$ & 4522.7 & 1380 \\
\hline \multirow{3}{*}{ Storm 2} & 0.08 & $17-22$ & 130.1 & 0.8 \\
\hline & 0.23 & $11-13,12-23,17-22$ & 408.0 & 29 \\
\hline & 0.38 & $8-9,8-10,11-13,12-13,12-23,17-22,21-22$ & 1771.8 & 820 \\
\hline \multirow{3}{*}{ Storm 3} & 0.08 & $\mathrm{NO}$ & 0 & 0.5 \\
\hline & 0.23 & $2-6,3-9,8-9$ & 22.4 & 56 \\
\hline & 0.38 & $1-3,2-4,2-6,3-9,8-9,8-10$ & 737.0 & 221 \\
\hline \multirow{3}{*}{ Storm 4} & 0.08 & NO & 0 & 0.8 \\
\hline & 0.23 & NO & 0 & 0.7 \\
\hline & 0.38 & $1-3,3-9$ & 350.0 & 4 \\
\hline \multirow{3}{*}{ Storm 5} & 0.08 & $17-22$ & 130.1 & 5 \\
\hline & 0.23 & $11-13,12-23,17-22$ & 390.3 & 61 \\
\hline & 0.38 & $1-5,2-6,3-9,6-10,11-3,12-13,12-23,17-22$ & 2188.0 & 1045 \\
\hline
\end{tabular}

The last column of Table VI reports the computational times required for solving the corresponding MILP models. It is shown that the total computational time increases rapidly as the failure uncertainty budget $\Gamma$ increases. Indeed, a large value of $\Gamma$ represents a big failure uncertainty set $\mathbb{U}$, i.e., a large 
searching space for the optimization problem. However, small values of $\Gamma$ result in small sets of critical lines (candidates for protection) and are, therefore, probably more interesting to system managers who have to face limited investment budgets.

\section{DISCUSSION AND CONCLUSION}

Physical models for evaluating the impact of natural hazards on infrastructure networks are affected by many uncertainties, including the intensity model of the natural hazard, the components structural fragility model and the probabilistic model of the restoration time of the failed components. These uncertainties can lead to the unreliable identification of system vulnerabilities. On the other hand, basic attacker-defender interdiction models based on the worst-case analysis can lead to an excessively conservative vulnerability analysis for CI systems subject to natural hazards. This paper presents a novel optimization-based framework for the identification of CI vulnerabilities under natural hazards; system vulnerability is interpreted from the perspective of critical components analysis and focuses on the identification of important components (or combinations of components), whose failures have a large impact on system functionality. In this framework, the estimated probabilities of components failure and restoration under specific natural hazards are integrated into a bi-level attacker-defender game interdiction model. This approach bridges the gap between the difficulties of accurately predicting the failure probabilities of system components in the simulation-based models and the over-conservatism of the basic attacker-defender approaches for CI vulnerabilities analysis under natural hazards. Therefore, it provides a valuable way to help decision-makers in making informed pre-hazard hardening planning decisions.

By formulating the parameterized uncertainty set $\mathbb{U}$ of the component failure states subject to the hazard, we are able to control the conservatism of the optimization solution via the uncertainty budgets $\Gamma$ and $\Upsilon$. For example, a larger value of the failure uncertainty budget $\Gamma$ represents that the decision maker is more conservative (i.e., assuming a larger quantity of "bad luck") and the failure of a "reliable" line, i.e., having smaller failure probability $p_{l t}$, is even increasingly allowed. In other words, the decision maker may select a large $\Gamma$ if he/she believes that component failure probabilities are affected by large uncertainties. This approach allows the decision maker a level of flexibility in setting the tradeoff between robustness and performance.

Mathematically, the proposed model configures a bi-level max-min MILP, which is challenging to be solved directly. By leveraging the properties of the lower level LP problem, the model is transformed into an equivalent one-level MILP problem and, then, solved by an efficient global solver, i.e., CPLEX. The application to a case study involving the georeferenced RTS24 test system under simulated wind storms demonstrates the effectiveness of the proposed approach for the identification of $\mathrm{CI}$ vulnerabilities due to a specific hazard event. These identified critical locations, which are conditional on the specific hazard, can be provided to the decision maker for use in supporting short-term pre-event 
preparation practices, e.g. choosing critical power poles to be hardened or allocating backup power units in a power grid, before a specific wind storm strikes the system. By setting different values of the failure uncertainty budget $\Gamma$, the decision maker can select the sets of the most critical components to be hardened, which are compatible with the investment budget.

The present study considers binary states of system components, i.e., completely out of service and fully operational, which is a modeling approach widely adopted in literature (ㅅroyo, 2010; $\underline{\text { G. Brown }}$ et al., 2005; G. Brown et al., 2006; Y. Fang \& Sansavini, 2017; Ouyang \& Fang, 2017; Salmeron et al., 2004; Wood, 2011). However, it may be also interesting to consider multiple states of damage, e.g., a damaged overhead line is operational but with a reduced capacity. Moreover, due to the interconnected and dynamic nature of modern power systems, small disturbances may trigger long chains of knock-on component failures that can lead to massive power outages, i.e., the so-called cascading failures. The modeling of these is still a challenging problem, because of the many different mechanisms involved and the limited data available from historical cascade events (Y.-P. Fang, Pedroni, \& Zio, 2015; B. Li, Barker, \& Sansavini, 2017; Zio, 2016): for this reason they have not been taken into account in the present study and will be considered in future works.

Power systems operations are also affected by many uncertainties, e.g., the capacity of renewable power generators and the power demand. One possible way to take into account these uncertainties is to construct another uncertainty set of the related system parameters $\mathbb{U}^{*}$ in the same max-min optimization framework as the present study. Then, the optimization for the worst case scenario, i.e., for the worst case values of both the uncertain system parameters $\mathbb{U}^{*}$ and the uncertain component failure states $\mathbb{U}$, can be carried out within the first level problem in (8). This new problem can be solved by the same idea of Section 4, i.e., transforming it into a single-level maximization problem by considering the dual of the inner-level problem.

Finally, the exponential increase in computational time with the size of the problem is a common feature of branch-and-cut algorithms when addressing MIP problems (Y. Fang \& Sansavini, 2017; Ouyang \& Fang, 2017). Although current commercial companies routinely solve MIP problems involving millions of variables and hundreds of thousands of constraints, more sophisticated techniques might also be required to ensure the applicability of the proposed restoration planning problem to largescale power systems with thousands of nodes and lines. To this aim, the study of efficient solution methodologies for two-stage robust optimization is an active field of research in mathematical optimization (Bertsimas et al., 2011). Promising solution methods, such as the column-and-constraint decomposition (Zeng \& Zhao, 2013), can be applied to the proposed problem in our future study.

\section{ACKNOWLEDGEMENTS}

The authors are grateful to the anonymous referees for their useful comments and suggestions to the original version of this paper. 


\section{REFERENCE}

7th AIMMS-MOPTA Optimization Modeling Competition. (2015). Retrieved from http://coral.ie.lehigh.edu/ mopta2015/AIMMS_MOPTA_case_2015.pdf

Adachi, T., \& Ellingwood, B. R. (2008). Serviceability of earthquake-damaged water systems: Effects of electrical power availability and power backup systems on system vulnerability. Reliability Engineering \& System Safety, 93(1), 78-88.

Aerts, J. C., Lin, N., Botzen, W., Emanuel, K., \& de Moel, H. (2013). Low-Probability Flood Risk Modeling for New York City. Risk Analysis, 33(5), 772-788.

Apostolakis, G. E., \& Lemon, D. M. (2005). A screening methodology for the identification and ranking of infrastructure vulnerabilities due to terrorism. Risk Analysis, 25(2), 361-376.

Arroyo, J. M. (2010). Bilevel programming applied to power system vulnerability analysis under multiple contingencies. IET generation, transmission \& distribution, 4(2), 178-190.

Aven, T. (2011). On some recent definitions and analysis frameworks for risk, vulnerability, and resilience. Risk Analysis, 31(4), 515-522.

Batke, S. P., Jocque, M., \& Kelly, D. L. (2014). Modelling hurricane exposure and wind speed on a mesoclimate scale: a case study from Cusuco NP, Honduras. PloS one, 9(3), e91306.

Bayliss, C., Bayliss, C. R., \& Hardy, B. J. (2012). Transmission and distribution electrical engineering: Elsevier.

Ben-Tal, A., El Ghaoui, L., \& Nemirovski, A. (2009). Robust optimization: Princeton University Press.

Bertsimas, D., Brown, D. B., \& Caramanis, C. (2011). Theory and applications of robust optimization. SIAM review, 53(3), 464-501.

Bertsimas, D., \& Thiele, A. (2006). Robust and data-driven optimization: Modern decision-making under uncertainty.

Bhuiyan, M., \& Allan, R. (1994). Inclusion of weather effects in composite system reliability evaluation using sequential simulation. IEE Proceedings-Generation, Transmission and Distribution, 141(6), 575-584.

Bier, V. M., Gratz, E. R., Haphuriwat, N. J., Magua, W., \& Wierzbicki, K. R. (2007). Methodology for identifying near-optimal interdiction strategies for a power transmission system. Reliability Engineering \& System Safety, 92(9), 1155-1161.

Birkmann, J. (2007). Risk and vulnerability indicators at different scales: Applicability, usefulness and policy implications. Environmental Hazards, 7(1), 20-31.

Bjarnadottir, S., Li, Y., \& Stewart, M. G. (2012). Hurricane risk assessment of power distribution poles considering impacts of a changing climate. Journal of Infrastructure Systems, 19(1), 1224.

Booker, G., Torres, J., Guikema, S., Sprintson, A., \& Brumbelow, K. (2010). Estimating cellular network performance during hurricanes. Reliability Engineering \& System Safety, 95(4), 337344.

Brown, G., Carlyle, M., Salmeron, J., \& Wood, K. (2005). Analyzing the vulnerability of critical infrastructure to attack and planning defenses. Tutorials in Operations Research: Emerging Theory, Methods, and Applications, 102-123.

Brown, G., Carlyle, M., Salmerón, J., \& Wood, K. (2006). Defending critical infrastructure. Interfaces, 36(6), 530-544.

Brown, R. (2009). Cost-benefit analysis of the deployment of utility infrastructure upgrades and storm hardening programs. Quanta Technology, Raleigh.

Cadini, F., Agliardi, G. L., \& Zio, E. (2017a). Estimation of rare event probabilities in power transmission networks subject to cascading failures. Reliability Engineering \& System Safety, 158, 9-20.

Cadini, F., Agliardi, G. L., \& Zio, E. (2017b). A modeling and simulation framework for the reliability/availability assessment of a power transmission grid subject to cascading failures under extreme weather conditions. Applied Energy, 185, 267-279.

Campbell, R. J. (2012). Weather-related power outages and electric system resiliency. 
Canham, C. D., Papaik, M. J., \& Latty, E. F. (2001). Interspecific variation in susceptibility to windthrow as a function of tree size and storm severity for northern temperate tree species. Canadian Journal of Forest Research, 31(1), 1-10.

Church, R. L., Scaparra, M. P., \& Middleton, R. S. (2004). Identifying critical infrastructure: the median and covering facility interdiction problems. Annals of the Association of American Geographers, 94(3), 491-502.

Cutter, S. L., Ismail-Zadeh, A., Alcantara-Ayala, I., Altan, O., Baker, D. N., Briceno, S., . . McBean, G. A. (2015). Global risks: Pool knowledge to stem losses from disasters. Nature, 522, 277 279.

Davis, C., Wang, W., Chen, S. S., Chen, Y., Corbosiero, K., DeMaria, M., . . Michalakes, J. (2008). Prediction of landfalling hurricanes with the Advanced Hurricane WRF model. Monthly weather review, 136(6), 1990-2005.

Dawson, R. J., Peppe, R., \& Wang, M. (2011). An agent-based model for risk-based flood incident management. Natural Hazards, 59(1), 167-189.

Delgadillo, A., Arroyo, J. M., \& Alguacil, N. (2010). Analysis of electric grid interdiction with line switching. IEEE Transactions on power systems, 25(2), 633-641.

Dorst, N. (2017). What is the average forward speed of a hurricane. Retrieved from http://www.aoml.noaa.gov/hrd/tcfaq/tcfaqHED.html

Dueñas-Osorio, L., \& Vemuru, S. M. (2009). Cascading failures in complex infrastructure systems. Structural safety, 31(2), 157-167.

Duffey, R. B., \& Ha, T. (2013). The probability and timing of power system restoration. IEEE transactions on power delivery, 28(1), 3-9.

Espinoza, S., Panteli, M., Mancarella, P., \& Rudnick, H. (2016). Multi-phase assessment and adaptation of power systems resilience to natural hazards. Electric Power Systems Research, $136,352-361$.

Ezell, B. C. (2007). Infrastructure Vulnerability Assessment Model (I-VAM). Risk Analysis, 27(3), 571-583.

Fang, Y.-P., Pedroni, N., \& Zio, E. (2015). Optimization of Cascade-Resilient Electrical Infrastructures and its Validation by Power Flow Modeling. Risk Analysis, 35(4), 594-607.

Fang, Y.-P., \& Zio, E. (2019). Game-Theoretic Decision Making for the Resilience of Interdependent Infrastructures Exposed to Disruptions. In D. Gritzalis, M. Theocharidou, \& G. Stergiopoulos (Eds.), Critical Infrastructure Security and Resilience: Theories, Methods, Tools and Technologies (pp. 97-114): Springer International Publishing.

Fang, Y., \& Sansavini, G. (2017). Optimizing power system investments and resilience against attacks. Reliability Engineering \& System Safety, 159, 161-173. doi:http://dx.doi.org/10.1016/j.ress.2016.10.028

Fenton, G. A., \& Sutherland, N. (2011). Reliability-based transmission line design. IEEE Transactions on Power Delivery, 26(2), 596-606.

Füssel, H.-M. (2007). Vulnerability: a generally applicable conceptual framework for climate change research. Global environmental change, 17(2), 155-167.

Grigg, C., Wong, P., Albrecht, P., Allan, R., Bhavaraju, M., Billinton, R., . . Kuruganty, S. (1999). The IEEE reliability test system-1996. A report prepared by the reliability test system task force of the application of probability methods subcommittee. IEEE Transactions on power systems, 14(3), 1010-1020.

Guikema, S. D., Davidson, R. A., \& Liu, H. (2006). Statistical models of the effects of tree trimming on power system outages. IEEE Transactions on Power Delivery, 21(3), 1549-1557.

Guikema, S. D., Quiring, S. M., \& Han, S. R. (2010). Prestorm estimation of hurricane damage to electric power distribution systems. Risk Analysis, 30(12), 1744-1752.

Haimes, Y. Y. (2006). On the definition of vulnerabilities in measuring risks to infrastructures. Risk Analysis, 26(2), 293-296.

Han, S. R., Guikema, S. D., \& Quiring, S. M. (2009). Improving the predictive accuracy of hurricane power outage forecasts using generalized additive models. Risk Analysis, 29(10), 1443-1453.

Hangan, H., Savory, E., El Damatty, A., Galsworthy, J., \& Miller, C. (2008). Modeling and prediction of failure of transmission lines due to high intensity winds. Struct. Congr.

Hernandez-Fajardo, I., \& Dueñas-Osorio, L. (2011). Sequential propagation of seismic fragility across interdependent lifeline systems. Earthquake Spectra, 27(1), 23-43. 
Hernandez-Fajardo, I., \& Dueñas-Osorio, L. (2013). Probabilistic study of cascading failures in complex interdependent lifeline systems. Reliability Engineering \& System Safety, 111, 260272.

Holland, G. J. (1980). An analytic model of the wind and pressure profiles in hurricanes. Monthly weather review, 108(8), 1212-1218.

Holland, G. J., Belanger, J. I., \& Fritz, A. (2010). A revised model for radial profiles of hurricane winds. Monthly Weather Review, 138(12), 4393-4401.

Hong, L., Ouyang, M., Peeta, S., He, X., \& Yan, Y. (2015). Vulnerability assessment and mitigation for the Chinese railway system under floods. Reliability Engineering \& System Safety, 137, 58-68.

IBM. (2015). IBM ILOG CPLEX Optimization Studio Getting Started with CPLEX. Retrieved from

Jenelius, E., \& Mattsson, L.-G. (2012). Road network vulnerability analysis of area-covering disruptions: A grid-based approach with case study. Transportation research part A: policy and practice, 46(5), 746-760.

Johansson, J., Hassel, H., \& Zio, E. (2013). Reliability and vulnerability analyses of critical infrastructures: comparing two approaches in the context of power systems. Reliability Engineering \& System Safety, 120, 27-38.

Jönsson, H., Johansson, J., \& Johansson, H. (2008). Identifying critical components in technical infrastructure networks. Proceedings of the Institution of Mechanical Engineers, Part O: Journal of Risk and Reliability, 222(2), 235-243.

Kadri, F., Birregah, B., \& Châtelet, E. (2014). The impact of natural disasters on critical infrastructures: A domino effect-based study. Journal of Homeland Security and Emergency Management, 11(2), 217-241.

Kröger, W., \& Zio, E. (2011). Vulnerable systems: Springer Science \& Business Media.

Kwasinski, A. (2010). Analysis of vulnerabilities of telecommunication systems to natural disasters. Paper presented at the Systems Conference, 2010 4th Annual IEEE.

Li, B., Barker, K., \& Sansavini, G. (2017). Measuring Community and Multi-Industry Impacts of Cascading Failures in Power Systems. IEEE Systems Journal.

Li, G., Zhang, P., Luh, P. B., Li, W., Bie, Z., Serna, C., \& Zhao, Z. (2014). Risk analysis for distribution systems in the northeast US under wind storms. IEEE Transactions on power systems, 29(2), 889-898.

Li, Y., \& Ellingwood, B. R. (2006). Hurricane damage to residential construction in the US: Importance of uncertainty modeling in risk assessment. Engineering structures, 28(7), 10091018.

Lin, N., Emanuel, K., Oppenheimer, M., \& Vanmarcke, E. (2012). Physically based assessment of hurricane surge threat under climate change. Nature Climate Change, 2(6), 462-467.

Lindell, M. K., \& Prater, C. S. (2003). Assessing community impacts of natural disasters. Natural Hazards Review, 4(4), 176-185.

Liu, H., Davidson, R. A., \& Apanasovich, T. V. (2007). Statistical forecasting of electric power restoration times in hurricanes and ice storms. IEEE Transactions on power systems, 22(4), 2270-2279.

Matisziw, T. C., \& Murray, A. T. (2009). Modeling s-t path availability to support disaster vulnerability assessment of network infrastructure. Computers \& Operations Research, 36(1), $16-26$.

Matisziw, T. C., Murray, A. T., \& Grubesic, T. H. (2007). Bounding network interdiction vulnerability through cutset identification Critical Infrastructure (pp. 243-256): Springer.

Mohanpurkar, M., Sogbi, H. V., \& Suryanarayanan, S. (2015). Geographical Information Systems and Loop Flows in Power Systems Electric Power Engineering Research and Education (pp. 135153): Springer.

Munich, R., Kron, W., \& Schuck, A. (2014). Topics geo: Natural catastrophes 2013: Analyses, assessments, positions: Munchener Ruckversicherungs-Gesellschaft.

Murray, A. T., \& Grubesic, T. (2007). Critical infrastructure: reliability and vulnerability: Springer Science \& Business Media.

Na, U. J., \& Shinozuka, M. (2009). Simulation-based seismic loss estimation of seaport transportation system. Reliability Engineering \& System Safety, 94(3), 722-731. 
Nateghi, R., Guikema, S. D., \& Quiring, S. M. (2011). Comparison and validation of statistical methods for predicting power outage durations in the event of hurricanes. Risk Analysis, 31(12), 1897-1906.

NOAA. (2016). Tracking data of the Typhoon Meranti. Retrieved 2016 October http://ftp.emc.ncep.noaa.gov/wd20vxt/hwrf-init/decks/bwp162016.dat

Oh, E. H., Deshmukh, A., \& Hastak, M. (2012). Criticality assessment of lifeline infrastructure for enhancing disaster response. Natural Hazards Review, 14(2), 98-107.

Ouyang, M., \& Dueñas-Osorio, L. (2014). Multi-dimensional hurricane resilience assessment of electric power systems. Structural safety, 48, 15-24.

Ouyang, M., \& Fang, Y.-P. (2017). A Mathematical Framework to Optimize Critical Infrastructure Resilience against Intentional Attacks. Computer-Aided Civil and Infrastructure Engineering.

Panteli, M., Pickering, C., Wilkinson, S., Dawson, R., \& Mancarella, P. (2016). Power System Resilience to Extreme Weather: Fragility Modelling, Probabilistic Impact Assessment, and Adaptation Measures. IEEE Transactions on power systems.

Pidgeon, N. (2012). Climate change risk perception and communication: addressing a critical moment? Risk Analysis, 32(6), 951-956.

Poljanšek, K., Bono, F., \& Gutiérrez, E. (2012). Seismic risk assessment of interdependent critical infrastructure systems: the case of European gas and electricity networks. Earthquake Engineering \& Structural Dynamics, 41(1), 61-79.

Ramirez-Marquez, J. E. (2010). A bi-objective approach for shortest-path network interdiction. Computers \& Industrial Engineering, 59(2), 232-240.

Rocchetta, R., Li, Y., \& Zio, E. (2015). Risk assessment and risk-cost optimization of distributed power generation systems considering extreme weather conditions. Reliability Engineering \& System Safety, 136, 47-61.

Salman, A. M., Li, Y., \& Stewart, M. G. (2015). Evaluating system reliability and targeted hardening strategies of power distribution systems subjected to hurricanes. Reliability Engineering \& System Safety, 144, 319-333.

Salmeron, J., Wood, K., \& Baldick, R. (2004). Analysis of electric grid security under terrorist threat. Power Systems, IEEE Transactions on, 19(2), 905-912.

Savory, E., Parke, G. A., Zeinoddini, M., Toy, N., \& Disney, P. (2001). Modelling of tornado and microburst-induced wind loading and failure of a lattice transmission tower. Engineering Structures, 23(4), 365-375.

Scholz, R. W., Blumer, Y. B., \& Brand, F. S. (2012). Risk, vulnerability, robustness, and resilience from a decision-theoretic perspective. Journal of Risk Research, 15(3), 313-330.

Shannon, C. E. (2001). A mathematical theory of communication. ACM SIGMOBILE Mobile Computing and Communications Review, 5(1), 3-55.

Sohn, J. (2006). Evaluating the significance of highway network links under the flood damage: An accessibility approach. Transportation research part A: policy and practice, 40(6), 491-506.

Vickery, P. J., \& Skerlj, P. F. (2005). Hurricane gust factors revisited. Journal of Structural Engineering, 131(5), 825-832.

Wang, Y., Chen, C., Wang, J., \& Baldick, R. (2016). Research on resilience of power systems under natural disasters - a review. IEEE Transactions on power systems, 31(2), 1604-1613.

Winkler, J., Duenas-Osorio, L., Stein, R., \& Subramanian, D. (2010). Performance assessment of topologically diverse power systems subjected to hurricane events. Reliability Engineering \& System Safety, 95(4), 323-336.

Wood, R. K. (2011). Bilevel network interdiction models: Formulations and solutions. Wiley Encyclopedia of Operations Research and Management Science.

Xie, Q., \& Zhu, R. (2011). Earth, wind, and ice. IEEE Power and Energy Magazine, 9(2), 28-36.

Xu, L., \& Brown, R. E. (2008). A hurricane simulation method for Florida utility damage and risk assessment. Paper presented at the Power and Energy Society General Meeting-Conversion and Delivery of Electrical Energy in the 21st Century, 2008 IEEE.

Zapata, C., Silva, S., Gonzalez, H., Burbano, O., \& Hernandez, J. (2008). Modeling the repair process of a power distribution system. Paper presented at the Transmission and Distribution Conference and Exposition: Latin America, 2008 IEEE/PES.

Zeng, B., \& Zhao, L. (2013). Solving two-stage robust optimization problems using a column-andconstraint generation method. Operations Research Letters, 41(5), 457-461. 
Zio, E. (2016). Challenges in the vulnerability and risk analysis of critical infrastructures. Reliability Engineering \& System Safety, 152, 137-150.

Zio, E., \& Aven, T. (2011). Uncertainties in smart grids behavior and modeling: What are the risks and vulnerabilities? How to analyze them? Energy Policy, 39(10), 6308-6320.

Zio, E., \& Kroger, W. (2009). Vulnerability assessment of critical infrastructures. IEEE Reliability Society 2009 Annual Technology Report, 186.

Zio, E., \& Sansavini, G. (2011). Component criticality in failure cascade processes of network systems. Risk Analysis, 31(8), 1196-1210.

Zio, E., \& Sansavini, G. (2013). Vulnerability of smart grids with variable generation and consumption: A system of systems perspective. IEEE Transactions on Systems, Man, and Cybernetics: Systems, 43(3), 477-487. 